\title{
Impact of time-dependent nonaxisymmetric velocity perturbations on dynamo action of von Kármán-like flows
}

\author{
André Giesecke* and Frank Stefani \\ Institute of Fluid Dynamics, Helmholtz-Zentrum Dresden-Rossendorf, P. O. Box 510119, D-01314 Dresden, Germany \\ Javier Burguete \\ Departamento de Física y Matemática Aplicada, Universidad de Navarra, Irunlarrea 1, E-31008 Pamplona, Spain
}

(Received 6 August 2012; published 7 December 2012)

\begin{abstract}
We present numerical simulations of the kinematic induction equation in order to examine the dynamo efficiency of an axisymmetric von Kármán-like flow subject to time-dependent nonaxisymmetric velocity perturbations. The numerical model is based on the setup of the French von Kármán-sodium dynamo (VKS) and on the flow measurements from a water experiment conducted at the University of Navarra in Pamplona, Spain. The principal experimental observations that are modeled in our simulations are nonaxisymmetric vortexlike structures which perform an azimuthal drift motion in the equatorial plane. Our simulations show that the interactions of these periodic flow perturbations with the fundamental drift of the magnetic eigenmode (including the special case of nondrifting fields) essentially determine the temporal behavior of the dynamo state. We find two distinct regimes of dynamo action that depend on the (prescribed) drift frequency of an $(m=2)$ vortexlike flow perturbation. For comparatively slowly drifting vortices we observe a narrow window with enhanced growth rates and a drift of the magnetic eigenmode that is synchronized with the perturbation drift. The resonance-like enhancement of the growth rates takes place when the vortex drift frequency roughly equals the drift frequency of the magnetic eigenmode in the unperturbed system. Outside of this small window, the field generation is hampered compared to the unperturbed case, and the field amplitude of the magnetic eigenmode is modulated with approximately twice the vortex drift frequency. The abrupt transition between the resonant regime and the modulated regime is identified as a spectral exceptional point where eigenvalues (growth rates and frequencies) and eigenfunctions of two previously independent modes collapse. In the actual configuration the drift frequencies of the velocity perturbations that are observed in the water experiment are much larger than the fundamental drift frequency of the magnetic eigenmode that is obtained from our numerical simulations. Hence, we conclude that the fulfillment of the resonance condition might be unlikely in present day dynamo experiments. However, a possibility to increase the dynamo efficiency in the VKS experiment might be realized by an application of holes or fingers on the outer boundary in the equatorial plane. These mechanical distortions provoke an anchorage of the vortices at fixed positions thus allowing an adjustment of the temporal behavior of the nonaxisymmetric flow perturbations.
\end{abstract}

DOI: 10.1103/PhysRevE.86.066303

PACS number(s): 47.65.-d, 91.25.Cw, 52.65.Kj

\section{INTRODUCTION}

Cosmic magnetic fields are ubiquitous phenomena that are intrinsically coupled to most astrophysical objects like planets, stars, or galaxies. The origin of these fields involves the formation of electrical currents by means of a complex flow of a conducting fluid or plasma. This process, the so-called dynamo effect, is necessarily three dimensional and nonlinear, which makes an analytical or numerical approach difficult. Meanwhile, fluid-flow-driven generation of magnetic fields has also been obtained in laboratory experiments providing a complementary tool to astronomical observations or numerical simulations. However, whereas astrophysical dynamo action is comparably easy because of the large dimensions of the involved flows, its experimental realization requires considerable technical efforts [1]. An important obstacle for the occurrence of laboratory dynamo action arises from the scaling behavior of the power that is required to drive a flow with a requested magnetic Reynolds number, Rm. For turbulent flows this power scales $\propto \mathrm{Rm}^{3}$ so a reduction of the critical $\mathrm{Rm}$ for the

\footnotetext{
*a.giesecke@hzdr.de
}

onset of dynamo action is most important to achieve magnetic self-excitation at all.

So far, dynamo experiments based on a flow of a conducting fluid have been successfully conducted in Riga [2], Karlsruhe [3], and Cadarache [4]. The first two facilities made use of a more or less predetermined fluid flow essentially fixed by the forcing and the shape of the internal tubes. Note, however, that, at least in the Riga dynamo experiment, the saturation process involved a nontrivial back-reaction effect of the magnetic field that changes the geometry of the flow. Such effects might be even more pronounced in the Cadarache von Kármán-sodium (VKS) dynamo. In that experiment, the flow driving by two opposing impellers provides more freedom for the development of a saturated turbulent state, in which the back-reaction of the magnetic field on the fluid can strongly modify the geometry and dynamics of the flow. In an idealizing model the mean axisymmetric flow between counter-rotating impellers comprises two toroidal and two poloidal eddies (so-called $s 2 t 2$ topology), and it is well known that this flow is able to drive a dynamo [5,6]. Various attempts in different geometries have been made (numerically as well as experimentally) in order to examine dynamo action driven by such a flow [4,7-20]. However, so far, experimental dynamo 
action driven by a von Kármán-like flow is obtained only at the VKS facility and only when at least one of the flow-driving impellers is made of soft iron with a large relative permeability. Kinematic simulations of the Cadarache dynamo indicate a close linkage between the exclusive occurrence of dynamo action in the presence of soft iron impellers and the observed axisymmetry of the magnetic field [21,22]. Nevertheless, a fully satisfactory explanation of the working principle of this dynamo is still missing and it is still unclear whether the present experiments will ever be able to achieve growing equatorial dipole modes, which constitute the magnetic field geometry that has been expected from kinematic simulations with an axisymmetric flow field.

An improvement of present numerical models may require the explicit consideration of coherent nonaxisymmetric structures that repeatedly have been observed in water experiments using a von Kármán-like flow driving [23-25]. Nonaxisymmetric time-periodic flows with a dominant azimuthal wave number $m=2$ have also been found in 3D simulations of $s 2 t 2$ flows in spherical geometry [16]. Kinematic dynamo simulations using various manifestations of these velocity fields showed a surprising diversity of behavior patterns; however, self-generation of magnetic energy was found only when the time-dependent flow field was taken into account, whereas the simulations with the time-averaged flow or with different snapshots of the velocity field did not exhibit dynamo action. A similar behavior has also been found previously in an ideal two-dimensional model by the authors of Ref. [26], who examined the induction action of a uniform shear flow perturbed by a periodic variation on intermediate time scales. The authors found a perpetual amplification even for very small perturbation amplitudes and concluded that tiny distortions $\sim \mathrm{Rm}^{-1}$ can be sufficient to alter the ability of a flow to provide for dynamo action. This type of dynamo action has been attributed to non-normal growth in Ref. [27], where it was shown that an appropriate mixing of nonorthogonal eigenstates through a time-dependent linear operator can lead to growing modes even if the contributing eigenstates alone correspond to decaying solutions in a stationary system.

The main objective of our study is the behavior of the dynamo efficiency of a cylindrical VKS-like system subject to nonaxisymmetric velocity perturbations with a single azimuthal wave number $m=2$. Such velocity modes were observed in a water experiment conducted at the University of Navarra in Pamplona in order to analyze the influence of slowly evolving large-scale flow on the occurrence of dynamo action $[23,28]$. Here, we utilize the essential features of the measured flow field as the basis input for numerical simulations of the kinematic induction equation. Typical input parameters that are systematically varied are the flow amplitude (in terms of the magnetic Reynolds number) and the azimuthal drift motion of the implied nonaxisymmetric velocity perturbation. From the simulation data we extract the leading eigenmodes and the related eigenvalues in terms of growth rates and frequencies that describe field amplitude modulations and/or azimuthal field drift. Interestingly, for comparably low drift frequencies of the velocity perturbation, we, first, observe a phase locking of the magnetic eigenmode drift with the vortex drift which is replaced, for higher perturbation drift frequency, by the appearance of a time-modulated magnetic eigenmode. By analyzing the involved growth rates and frequencies in the phase-locked regime and in the modulated regime, we identify the transition between them as a spectral exceptional point where eigenvalues and eigenfunctions of two modes coincide $[29,30]$. The observed behavior is in close analogy with typical (resonant) mechanical systems subject to periodic forcing, like, e.g., spinning disk systems [31], or to the behavior observed in the stability study of water waves [32] and we will see, by analyzing the solution of a simple Mathieu equation, that the observed spectral structure is quite generic for systems under the influence of periodic forcing. Comparable effects have also been found in mean-field dynamos of $\alpha \omega$ type that were designed to explain the bisymmetric field pattern observed in spiral galaxies. In these models a periodic perturbation is caused by density waves due to spiraling arms, and a parametric resonance (also called swing excitation) is observed when the frequency of the perturbing velocity pattern is twice the oscillation frequency of the (axisymmetric) dynamo [33-37].

In the present paper, we show that a facilitation of dynamo action by periodic flow perturbations is also possible in more complex three-dimensional models that include magnetic diffusivity and potentially can be applied to existing dynamo experiments. In contrast to the dynamo models from Refs. [16,27] the observed increase of the growth rate occurs already without involving time-periodic states, which makes an interpretation in terms of non-normal growth (as in Ref. [27]) rather implausible.

\section{EXPERIMENTAL BACKGROUND}

The simulations presented below are motivated from a water experiment that is described in detail in Ref. [23]. In the experiment a von Kármán swirling flow is driven by two counterrotating impellers located at the end caps of a cylindrical vessel of radius $R=10 \mathrm{~cm}$ and height $H=20 \mathrm{~cm}$ [Fig. 1(a)].

Both impellers spin with a rotation rate up to $12 \mathrm{~Hz}$ so the resulting flow is highly turbulent with flow fluctuations of the same order as the mean flow [a typical snapshot of the turbulent flow is shown in Fig. 1(b)]. The velocity field is measured using laser Doppler velocimetry (LDV) and the mean velocity field is obtained by averaging the instantaneous flow for (at least) 100 impeller turns. The resulting axisymmetric velocity field consists of two toroidal cells and two meridional recirculating cells that are roughly restricted to each cylindrical half-space [Fig. 1(c)]. Comparable structures have been also observed in water experiments with similar configurations [24,25].

The cylindrical configuration combined with the specific flow driving imposes a discrete symmetry: The most symmetric flow that can be obtained between two propellers rotating in opposite directions breaks the reflection symmetry on any plane that contains the cylinder axis but preserves the symmetry about a $\pi$ rotation around any diameter in the equatorial plane [Fig. 2(a)]. However, in the water experiment, this symmetry is broken for $\operatorname{Re} \gtrsim 10^{4}$ (without internal symmetrizing fixtures) and the measured mean velocity field becomes asymmetric even when both impellers spin with equal rotation rates [23]. As a consequence, one of the cells [blue (upper cell) or red (lower cell) in Fig. 2(b)] becomes larger than the other. The averaged velocity field is meta-stable, and spontaneous jumps of the dominant cell from one side to the 


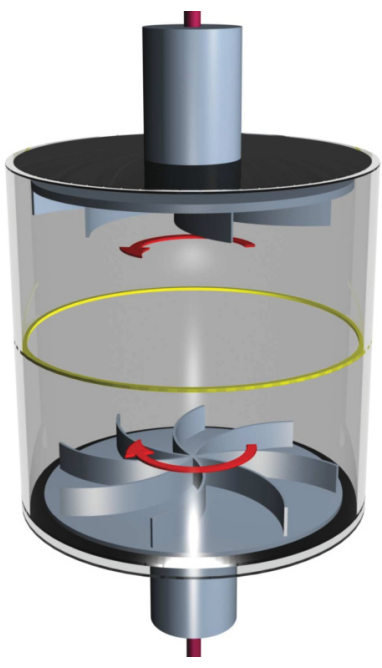

(a)

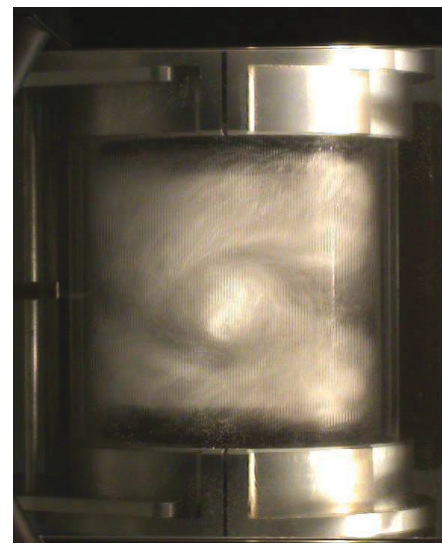

(b)

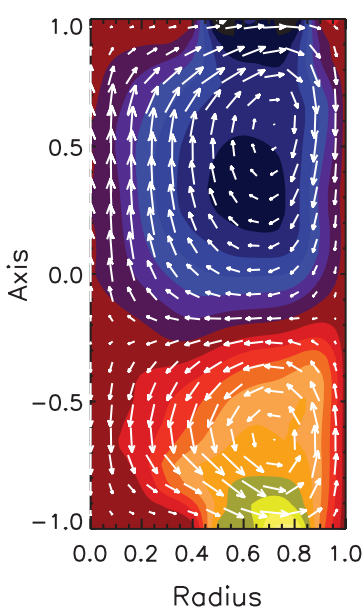

(c)

FIG. 1. (Color) (a) Sketch of the experimental setup. In the experiment the von Kármán swirling flow is driven by two counter-rotating impellers located at the end caps of a cylindrical vessel of radius $R=10 \mathrm{~cm}$ and height $H=20 \mathrm{~cm}$. The yellow ring denotes the equatorial plane of the cylinder. (b) Instantaneous snapshot of the turbulent fluid flow. Note the vortexlike structure roughly in the center of the vessel. (c) Mean axisymmetric flow field measured in the experiment. The colored structure denotes the toroidal flow $\left(u_{\varphi}\right)$ and the arrows denote the poloidal flow $\left(u_{r}, u_{z}\right)$. Although both impellers rotate with the same velocity different sizes of the flow cells occur due to spontaneous symmetry breaking.

other are observed (so-called inversions) with both possible states occurring with equal probability.

The transition time for an inversion is $\tau_{\text {inv }} \approx 10 \mathrm{~s}$ and the time between consecutive events varies from minutes to hours following a probability distribution $\rho(t)=\frac{1}{T_{0}} e^{-t / T_{0}}$ with a parameter $T_{0}$ determined by the noise intensity. $T_{0}$ decreases for increasing Reynolds number, i.e., inversions occur more frequently if the turbulence is more vigorous. The inversions of the velocity field introduce a slow time scale at which the large scale flow and hence the large scale magnetic field change their structure. In the long run, the enhanced dissipation arising from the transition between different eigenstates (corresponding to the different velocity fields) can result in a reduced efficiency for the dynamo process when the mean lifetime of a velocity state becomes of the same order as the magnetic diffusion time [38].
In addition to breaking of the equatorial symmetry the observed mean flow also violates the ideal axial symmetry. Coherent nonaxisymmetric structures emerge close to the equatorial plane (even before a turbulent state is reached) and establish a local swirling flow around an axis perpendicular to the main symmetry axis of the cylinder. The nonaxisymmetric flow perturbation is dominated by an azimuthal wave number $m=2$ and undergoes an azimuthal drift that is immediately linked to the flow orientation of the dominating toroidal cell. The drift frequency of the vortexlike pattern is related to the maximum azimuthal mean flow velocity $u_{\varphi}^{\max }$ by $\omega_{\mathrm{v}} \approx$ $0.3 u_{\varphi}^{\max } / R_{\mathrm{v}}$, where $R_{\mathrm{V}}$ denotes the radius of the maximum vortex velocity $\left(R_{\mathrm{v}} \approx 0.857\right.$ in units of the cylinder radius). The midsize structures provide an additional source of helicity, so it is likely that the vortices are involved in the dynamo process when a conducting fluid like liquid sodium is utilized.

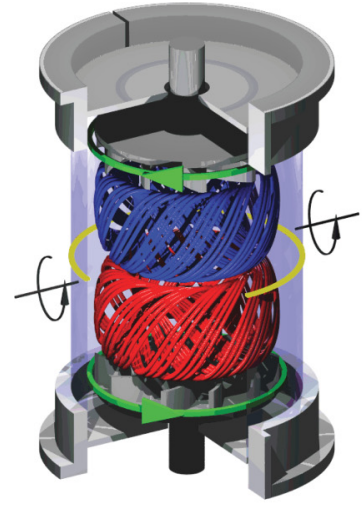

(a)

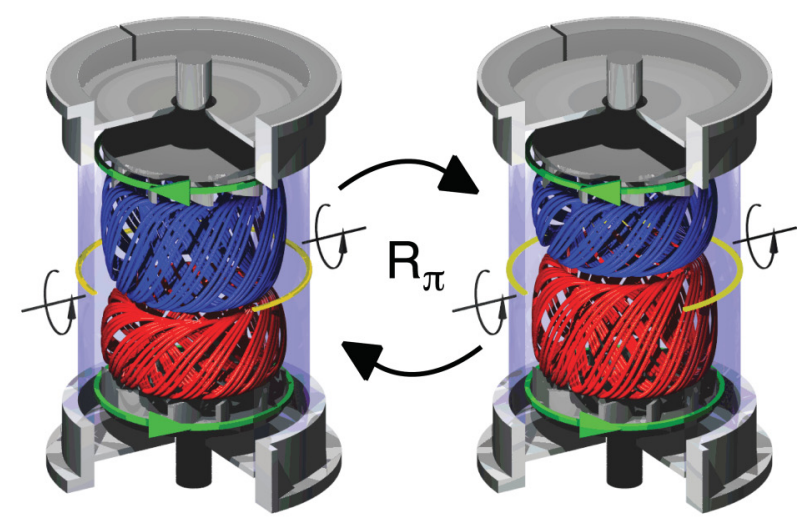

(b)

FIG. 2. (Color online) Reconstruction of averaged trajectories in the experiment. The blue (upper) and red (lower) streamlines represent the averaged trajectories impelled by each one of the top and bottom impellers. (a) Symmetric flow configuration. (b) Equatorial symmetry breaking observed in the experiment when $\mathrm{Re}>10^{4}$. Spontaneous jumps between both possible states are observed in the experiment. 


\section{NUMERICAL MODEL}

The temporal development of the magnetic flux density $\boldsymbol{B}$ induced from a flow $\boldsymbol{u}$ of a conducting fluid is described by the magnetic induction equation that results from the combination of Faraday's law, Ohm's law, and Ampère's law (without displacement current):

$$
\frac{\partial \boldsymbol{B}}{\partial t}=\boldsymbol{\nabla} \times(\boldsymbol{u} \times \boldsymbol{B}-\eta \boldsymbol{\nabla} \times \boldsymbol{B}) .
$$

In Eq. (1) $\eta$ denotes the magnetic diffusivity which is related to the electrical conductivity $\sigma$ and the vacuum permeability $\mu_{0}$ by $\eta=\left(\mu_{0} \sigma\right)^{-1}$. Equation (1) is time stepped, applying a finite volume method where a constraint transport scheme ensures the exact treatment of the solenoidal property of $\boldsymbol{B}$ (if the initial field is divergence free). Insulating boundary conditions are treated with a modified boundary integral equation approach which yields the tangential field components on the boundary from the normal field components on the whole surface of the computational domain $[39,40]$.

\section{A. The axisymmetric velocity field}

We use an analytically prescribed flow field that incorporates the main characteristics of a von Kármán flow and allows a convenient variation of the equatorial symmetry breaking. Since the typical lifetime of the mean flow state is much longer than the time scales governing the (nonaxisymmetric) flow perturbations, the dynamics introduced by the inversions can be ignored so we assume a time-independent axisymmetric velocity field. Equatorial symmetry breaking is modeled using a basic flow that is composed of two different axisymmetric parts with different symmetry properties. The main part is symmetric with respect to a $\pi$ rotation around any diameter in the equatorial plane. This contribution will be called even flow [Fig. 3(a)]. The geometric structure of the even flow consists of two counteroriented toroidal cells and two recirculating poloidal cells located in each cylindrical halfspace. Mathematically, the even part is prescribed by the so-called MND flow first proposed by Marié, Normand, and Daviaud [41],

$$
\begin{aligned}
& u_{r}^{\mathrm{e}}(r, z)=-\frac{\pi}{H} r(1-r)^{2}(1+2 r) \cos \left(\frac{2 \pi z}{H}\right), \\
& u_{\varphi}^{\mathrm{e}}(r, z)=4 \epsilon r(1-r) \sin \left(\frac{\pi z}{H}\right), \\
& u_{z}^{\mathrm{e}}(r, z)=(1-r)\left(1+r-5 r^{2}\right) \sin \left(\frac{2 \pi z}{H}\right),
\end{aligned}
$$

where $\epsilon$ represents the relation between toroidal and poloidal flow (here $\epsilon=0.8155$ ) and $H$ is the total height of the cylinder (here $H=2.0$ ).

The second contribution changes its sign when performing the same $\pi$ transformation and is called odd flow [Fig. 3(b)]. This contribution is determined by a single poloidal cell that spreads through the whole cylinder and a global azimuthal rotation $u_{\varphi}$ (independent of $z$ and $\varphi$ ). The odd flow contribution assumes the same expressions for $\epsilon, H$ and for the radial profile as used for the even flow (2) and is given by

$$
\begin{aligned}
& u_{r}^{\mathrm{o}}=\frac{\pi}{2 H} r(1-r)^{2}(1+2 r) \sin \left(\frac{2 \pi z}{H}\right), \\
& u_{\varphi}^{\mathrm{o}}=\epsilon r(1-r), \\
& u_{z}^{\mathrm{o}}=(1-r)\left(1+r-5 r^{2}\right) \cos ^{2}\left(\frac{\pi z}{H}\right) .
\end{aligned}
$$

The variation of the equatorial symmetry breaking is realized by multiplying the odd flow contribution (3) with a weighting factor $a \in[0 ; 1]$ so the total flow field is given by

$$
\boldsymbol{u}=\boldsymbol{u}^{e}+a \boldsymbol{u}^{o} .
$$

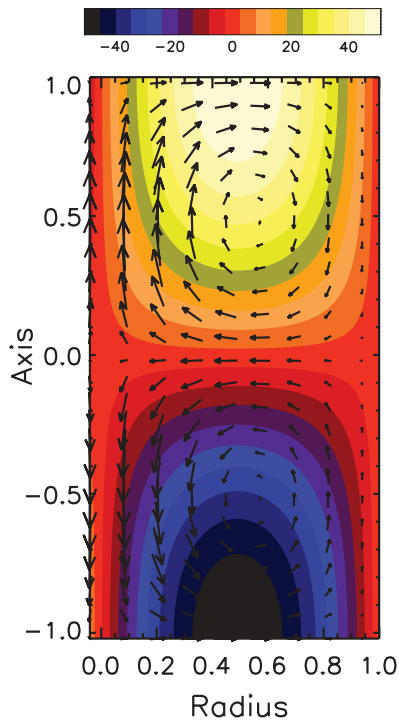

(a)

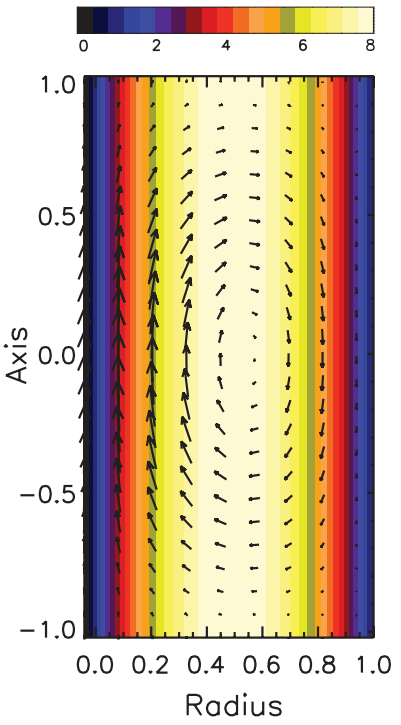

(b)

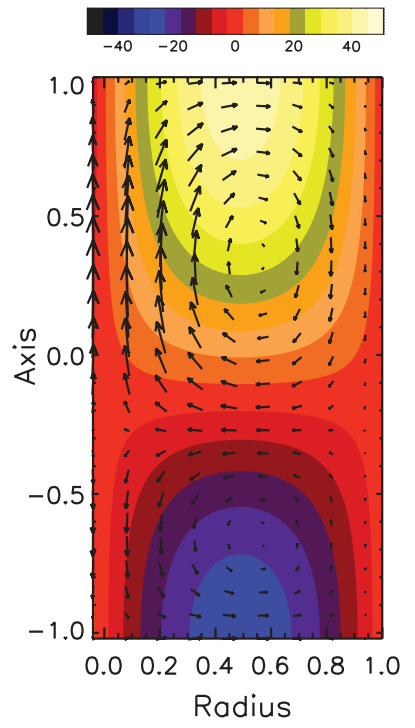

(c)

FIG. 3. (Color online) Prescribed velocity field. From left to right: (a) even flow (MND flow) as given by Eq. (2), (b) odd flow contribution given by Eq. (3), (c) total flow $\boldsymbol{u}=\boldsymbol{u}^{\mathrm{e}}+a \boldsymbol{u}^{\mathrm{o}}$ for $a=0.35$. The color-coded (gray shaded) pattern represents the azimuthal flow $\left(u_{\varphi}\right)$ and the arrows denote orientation and magnitude of the meridional flow $\left(u_{r}, u_{z}\right)$. 
$\boldsymbol{u}$ describes a flow field with two different cell sizes with the even contribution always being dominant [see Fig. 3(c)]. In our model the dominant toroidal eddy is always located close to the north (top) impeller (at $z=+H / 2$ ) and is characterized by a counterclockwise oriented azimuthal velocity [Fig. 3(c)].

The combined flow field is scaled allowing a systematically variation of the (prescribed) magnetic Reynolds number defined as

$$
\mathrm{Rm}=\frac{\mathcal{U R}}{\eta},
$$

where $\eta$ denotes the magnetic diffusivity, $\mathcal{R}$ a characteristic length scale (here $\mathcal{R}=1$, the radius of the cylindrical domain), and $\mathcal{U}$ is the peak velocity defined by $\mathcal{U}=U^{\max }=\max \left[\left(u_{r}^{2}+\right.\right.$ $\left.\left.u_{\varphi}^{2}+u_{z}^{2}\right)^{\frac{1}{2}}\right]$.

The flow field (4) is qualitatively similar to the model flow applied in Ref. [42], where dynamo action is examined in a VKS-like configuration with impellers rotating at different speeds. There are various other possibilities to realize the equatorial symmetry breaking of the ideal flow field (2) and it turns out that not every property of the observed flow field can always be reproduced exactly. For example, the definition of the flow field (4) does allow an easy adjustment of the symmetry breaking in terms of the parameter $a$ but exhibits different azimuthal velocities near the impellers. In this sense, the idealized flow (4) differs from the observed flow, which results from a forcing through impellers that are in exact counter-rotation, so the flow velocities close to both impellers are the same. Such deviations slightly influence the quantitative outcome of the simulations like, e.g., the critical magnetic Reynolds number for the onset of dynamo action;
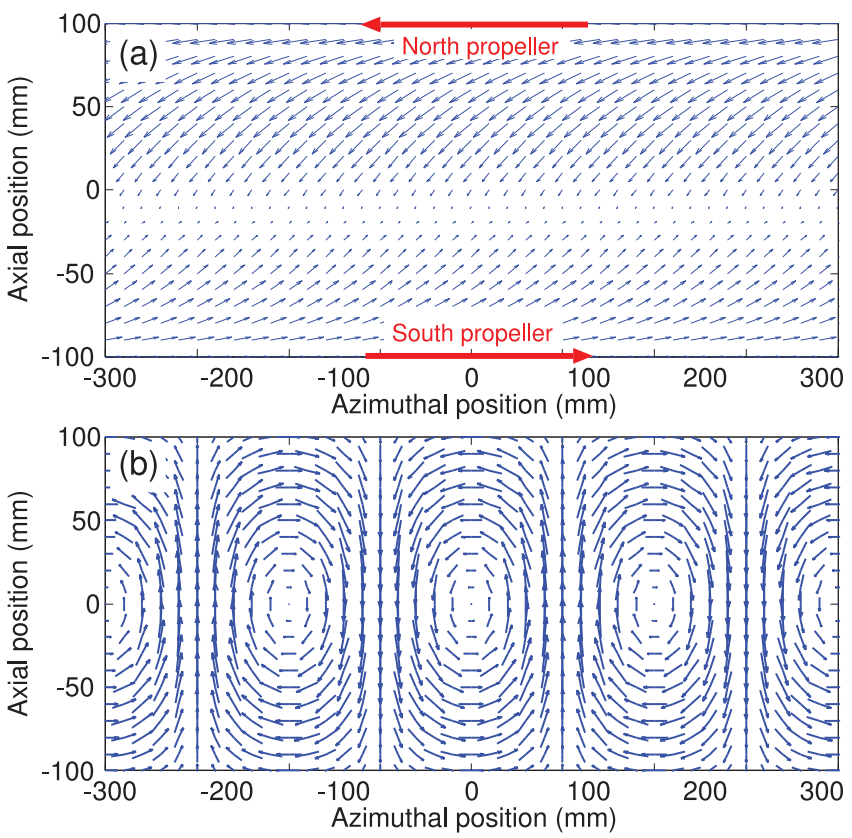

nevertheless, they do not affect the essential conclusions that will result from our simulations.

\section{B. Modeling of the nonaxisymmetric velocity perturbations}

The coherent nonaxisymmetric flow perturbations are located near the outer wall of the cylinder and close to the equatorial plane where a strong shear layer emerges that is caused by the opposite azimuthal flow orientation in each cylindrical half. The vortices allow a relaxation of the shear in the equatorial layer in some way similar to a Kelvin-Helmholtz instability but under turbulent conditions. The formation of the vortices in the experiment and the corresponding implementation in the numerical model are sketched in Fig. 4.

In the simulations, the vortices are parameterized by analytical expressions whereby the essential properties-the azimuthal wave number, thickness, diameter, and average radial position-are taken from the measurements. The center of the vortices with a typical diameter of 0.5 (and an aspect ratio of unity) is located around the equatorial plane with a maximum at a radius of $R_{\mathrm{v}}=0.857$ (all numbers are denoted in units of the cylinder radius $R=10 \mathrm{~cm}$ ). The nonaxisymmetric contribution of the vortices to the velocity field is explicitly given by

$$
\begin{aligned}
& u_{r}^{\mathrm{v}}=0, \\
& u_{\varphi}^{\mathrm{v}}=\frac{2 V_{\mathrm{m}}}{m} \cos \left[m\left(\varphi+\omega_{\mathrm{v}} t\right)\right] r^{2}(1-r) e^{-\left[\left(r-r_{0}\right) / \sigma\right]^{2}} \sin (4 z), \\
& u_{z}^{\mathrm{v}}=-2 V_{\mathrm{m}} \sin \left[m\left(\varphi+\omega_{\mathrm{v}} t\right)\right] r^{2}(1-r) e^{-\left[\left(r-r_{0}\right) / \sigma\right]^{2}} \cos ^{2}(2 z),
\end{aligned}
$$
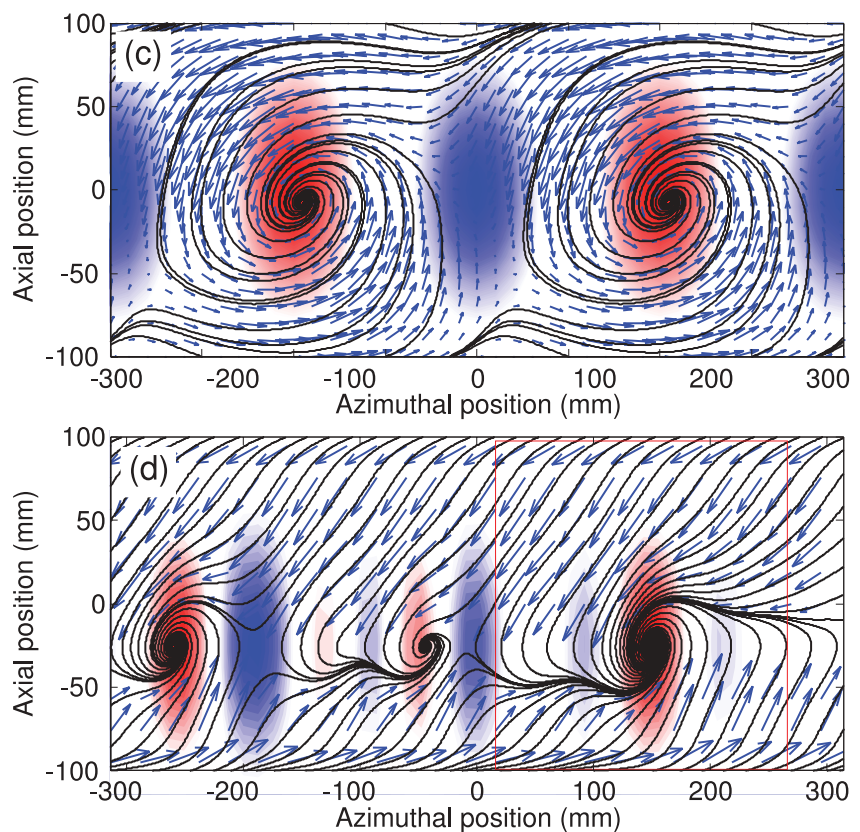

FIG. 4. (Color online) Mean flow and vortex pattern realized in the model: The figures show axisymmetric and nonaxisymmetric contributions of the velocity field in the $(\varphi, z)$ plane at $r=0.9$. Only the azimuthal and axial components of the velocity field are presented. (a) Odd and even axisymmetric part [Eqs. (2) and (3)]. (b) Vortices according to Eq. (6) for $m=2$. (c) Total velocity field [combining Eqs. (2), (3), and (6)] for $V_{\mathrm{m}}=0.2$. The colored (gray shaded) structures represent the radial component of the vorticity of the nonaxisymmetric contribution $\left(\nabla \times \boldsymbol{u}^{\mathrm{v}}\right)_{r}$ [red (dark gray) respectively blue (light gray) corresponds to positive repectively negative vorticity]. (d) Same as in (c) for the experimental data. 
with the axial coordinate $z$ restricted to the interval $z \in$ $[-\pi / 4 ; \pi / 4]$. In Eq. (6) $V_{\mathrm{m}}$ denotes the magnitude of the nonaxisymmetric perturbation and the quantities $\sigma$ and $r_{0}$ are estimated from the experimental observations (here always $r_{0}=0.9$ and $\sigma=0.12$ ). The azimuthal drift frequency of the vortex perturbation is denoted by $\omega_{\mathrm{v}}$, which in the experiment is linked to the azimuthal velocity of the dominant mean flow cell. In the system of units applied in the simulations the observed vortex drift frequency $\sim 0.3 u_{\varphi}^{\max } / R_{\mathrm{V}}$ corresponds to $\omega_{\mathrm{v}} \approx 17.1(14.9,14.0,13.1)$ for $a=0(0.62,0.84,1.00)$. Note that the drift frequency of the nonaxisymmetric velocity pattern with an azimuthal wave number $m$ is related to the previously defined vortex frequency by $\Omega_{\mathrm{p}}=m \omega_{\mathrm{v}}$. In the water experiment the vortices exhibit an intermittent behavior, e.g., sudden jumps to a state with a different wave number ( $m=3$ or $m=4$ ) or fluctuations of the vortex drift. Furthermore, the locations of the vortices undergo variations that are connected to the largest fluctuations of the mean velocity field. Those fluctuations appear centered around the shear layers and close to the wall. These temporal alterations are not considered in our numerical model, where we restrict our examinations to the case $m=2$ which corresponds to the most probable configuration observed in the experiment. In all cases the disturbance introduced by the nonaxisymmetric component remains only weak and has no influence on the actual $\mathrm{Rm}$ given by the maximum of the modulus of $\boldsymbol{u}$.

\section{RESULTS}

In the kinematic approach, the back-reaction of the magnetic field on the flow is neglected so the solution of the induction Eq. (1) represents a linear problem, which in principle could be solved with the ansatz

$$
\boldsymbol{B}(\boldsymbol{r}, t)=\boldsymbol{B}_{0}(\boldsymbol{r}) e^{\lambda t} .
$$

A dynamo solution is obtained if the magnetic energy density $E_{\text {mag }}=\left(2 \mu_{0}\right)^{-1}|\boldsymbol{B}|^{2}$ grows exponentially, $\propto e^{2 \lambda t}$. In general, $\lambda$ is a complex quantity, $\lambda=\gamma+i \omega$, where $\gamma$ denotes the field amplitude growth rate and $\omega$ denotes an oscillation or drift frequency. In case of a time-dependent velocity perturbation with a (given) period $T$, the general form of the solution differs from Eq. (7) and the proper time dependence follows from Floquet theory,

$$
\boldsymbol{B}(\boldsymbol{r}, t) \sim \sum_{i} e^{\mu_{i} t} \boldsymbol{P}_{i}(\boldsymbol{r}, t)
$$

where $\boldsymbol{P}_{i}(\boldsymbol{r}, t)=\boldsymbol{P}_{i}(\boldsymbol{r}, t+T)$ has period $T$ and $\rho_{i}=e^{\mu_{i} t}$ are the so-called Floquet multipliers (e.g., Refs. [43,44]). In the following, the growth rates represent magnetic field amplitude growth rates for the $(m=1)$ mode and the applied time scale is given by the magnetic diffusion time $\tau_{\eta}=R^{2} / \eta$. In accordance with Cowling's antidynamo theorem, dynamo solutions generated by a prescribed mean axisymmetric flow necessarily yield a nonaxisymmetric field. In all simulations presented below the $(m=1)$ mode is the dominant field contribution so the magnetic eigenmode behaves $\propto \cos \varphi$ and represents a dipole oriented along the equatorial plane.

\section{A. Equatorial symmetry breaking}

For a sufficiently large magnetic Reynolds number, the flow field (4) drives a dynamo with a typical structure shown in Fig. 5, which displays the eigenmodes for the undisturbed flow field ( $a=0)$ and the model case $a=0.62$. In both cases, the geometry is dominated by two interleaved banana-cell-like structures. However, in case of equatorial symmetry breaking ( $a=0.62$; Fig. 5 , right panel), a slight concentration of magnetic energy is observed in the upper cylindrical half-space containing the dominant flow cell. The breaking of the ideal equatorial symmetry suppresses dynamo action: When a symmetry-breaking flow contribution is added, the critical magnetic Reynolds number for the onset of dynamo action roughly increases $\propto a^{2}$ from $\mathrm{Rm}^{\mathrm{c}} \approx 50.5$ for $a=0$ to $\mathrm{Rm}^{\mathrm{c}}=109.5$ at $a=1$ [Figs. 6(a) and 6(b)].
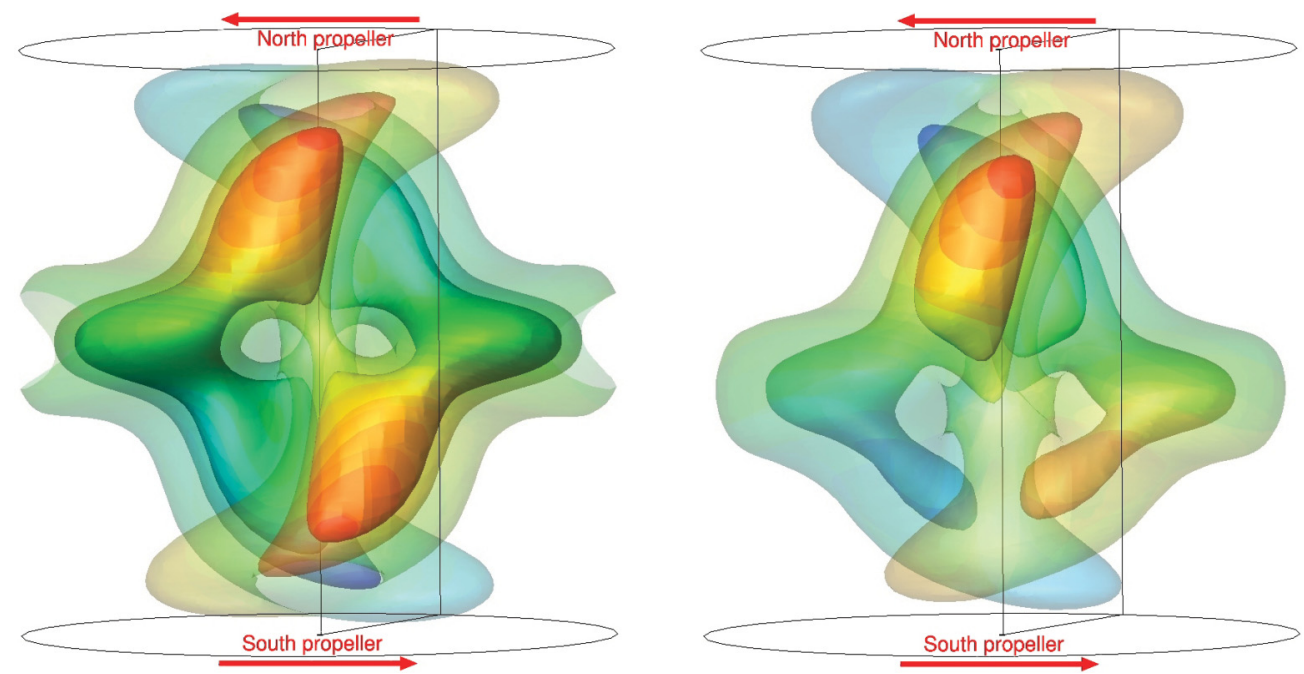

FIG. 5. (Color) Geometric structure of the dynamo eigenmode. Left: No equatorial symmetry breaking $(a=0)$, Rm $=70$. Right: $a=$ $0.62, \mathrm{Rm}=70$. The isosurfaces show the magnetic energy density at $10 \%, 30 \%$, and $50 \%$ of the respective maximum value. The colored contours denote the azimuthal magnetic field $B_{\varphi}$. Note the magnetic energy concentration in the upper part of the cylinder, which contains the dominant flow cell in case of equatorial symmetry breaking (right panel). 
(a)

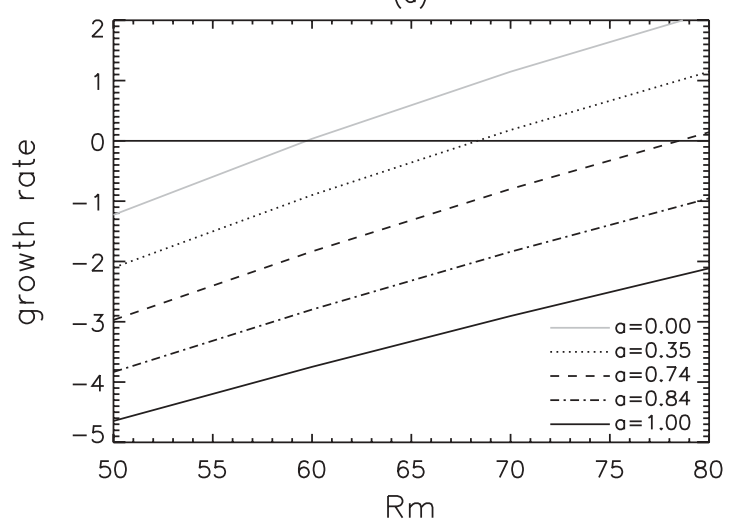

(b)

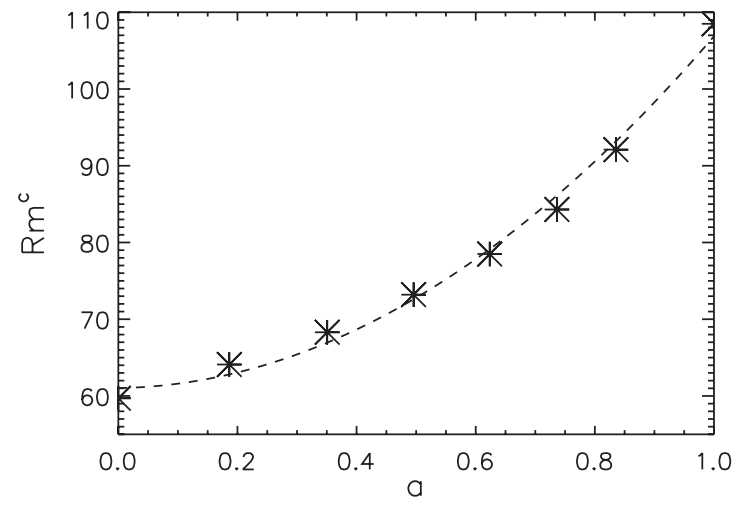

FIG. 6. (a) Growth rate versus Rm for various equatorial symmetry breaking $a$; (b) critical magnetic Reynolds number versus $a$. The dashed curve denotes a fit by a polynomial of degree 2 .

In the ideal symmetric case $(a=0)$, the eigenmode shows no time dependence except the exponential decay. With symmetry breaking, the $(m=1)$ mode exhibits an azimuthal drift around the cylinder axis, i.e., $\boldsymbol{B} \sim \boldsymbol{B}_{0}(r, z) e^{\lambda t} \cos (\varphi-$ $\left.\omega_{\mathrm{f}} t\right)$, with the magnetic field drift frequency $\omega_{\mathrm{f}}$ increasing nearly linearly with the degree of asymmetry $a$ and with Rm [Figs. 7(a) and 7(b)]. $\omega_{\mathrm{f}}$ is constant in time and is oriented opposite to the azimuthal flow in the dominant cell. For axisymmetric velocity fields [as $\boldsymbol{u}^{\mathrm{e}}$ and $\boldsymbol{u}^{\mathrm{o}}$, Eqs. (2) and (3)] poloidal and toroidal velocity components are decoupled, and their shear layer may have independent locations. The relative position of these shear layers controls the magnitude (and orientation) of the magnetic field drift. For example, an exclusive increment of the odd azimuthal flow contribution (which only shifts the toroidal shear layer) would alter the drift frequency. The change might be quite dramatic and even a reversal of the drift orientation is possible with the eigenmode corotating with the dominant azimuthal flow, as observed, for example, in Ref. [8]. A systematic study of the influence of this effect is underway.

\section{B. Impact of nonaxisymmetric velocity perturbations}

The presence of nonaxisymmetric velocity components results in a coupling between different azimuthal magnetic

(a)

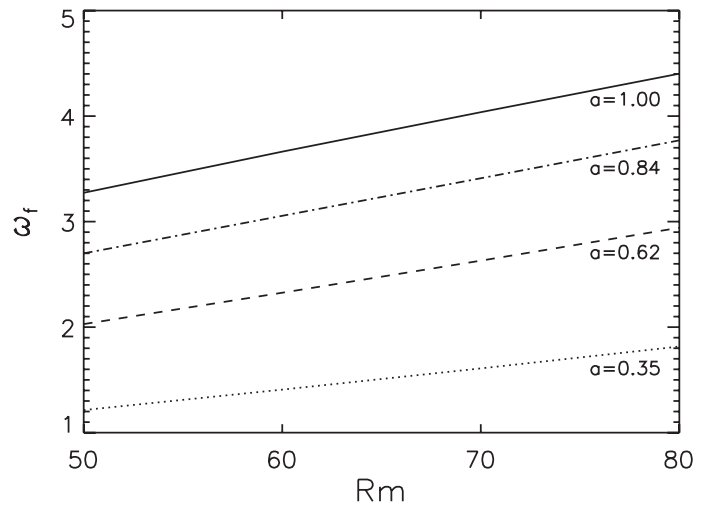

field modes that are separated in the purely axisymmetric problem. Hence, the characterization of the dynamo eigenmode with a single azimuthal wave number is no longer correct because (in principle) all azimuthal wave numbers are linked. In the following, we examine the impact of a nonaxisymmetric flow perturbation with a (single) wave number $m=2$ so two classes of magnetic eigenmodes arise which incorporate even azimuthal wave numbers $(m=0,2,4, \ldots)$ or odd azimuthal wave numbers $(m=1,3,5, \ldots)$, respectively. In our model, only the second class with odd wave numbers is relevant, whereas the even modes are not relevant for our problem because they decay on a faster time scale.

In the following, we keep the amplitude of the nonaxisymmetric velocity contribution fixed at $V_{\mathrm{m}}=0.3 U^{\mathrm{max}}$, which roughly corresponds to the value observed in the experiments. In this regime the presence of the nonaxisymmetric velocity component neither changes the global (axisymmetric) flow topology nor the actual magnetic Reynolds number. We further assume that the drift frequency of the vortex, $\omega_{\mathrm{v}}$, is a free parameter that is systematically varied in the interval $\omega_{\mathrm{v}} \in$ $[-60,+60]$. The maximum or minimum value correspond to a vortex drift velocity approximately equal to the maximum azimuthal flow velocity (recall that in the water experiment the observed vortex drift velocity is roughly $\left.0.3 u_{\varphi}^{\max }\right)$. We also examined the artificial case with a vortex drift orientation (b)

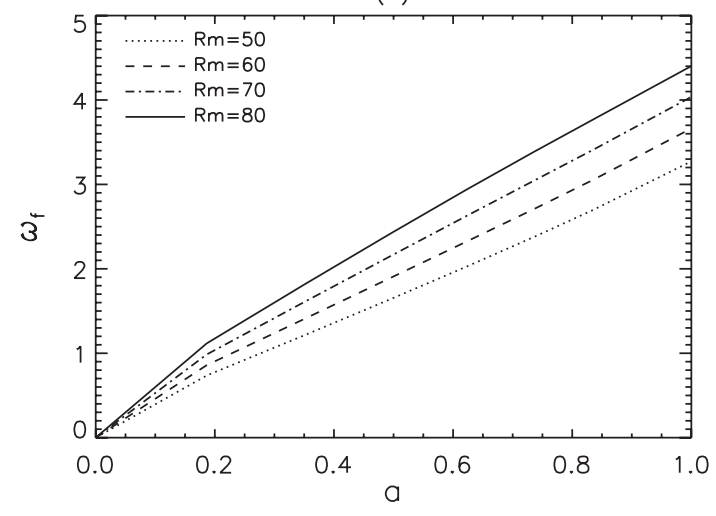

FIG. 7. (a) Drift frequency of the $(m=1)$ mode versus Rm for various $a$. (b) Drift frequency of the $(m=1)$ mode versus equatorial symmetry breaking $a$. Note the slight deviation from the linear behavior for small $a$. 
(a)

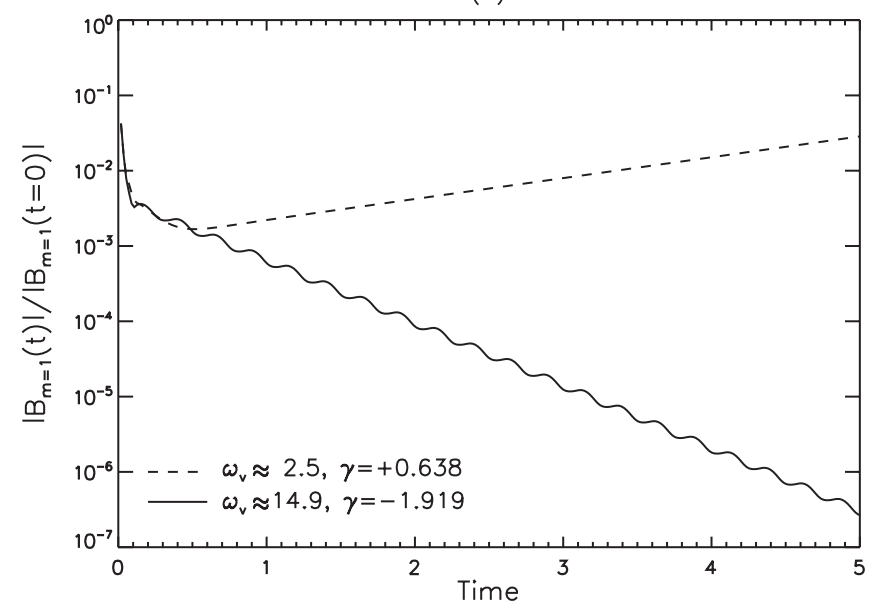

(b)

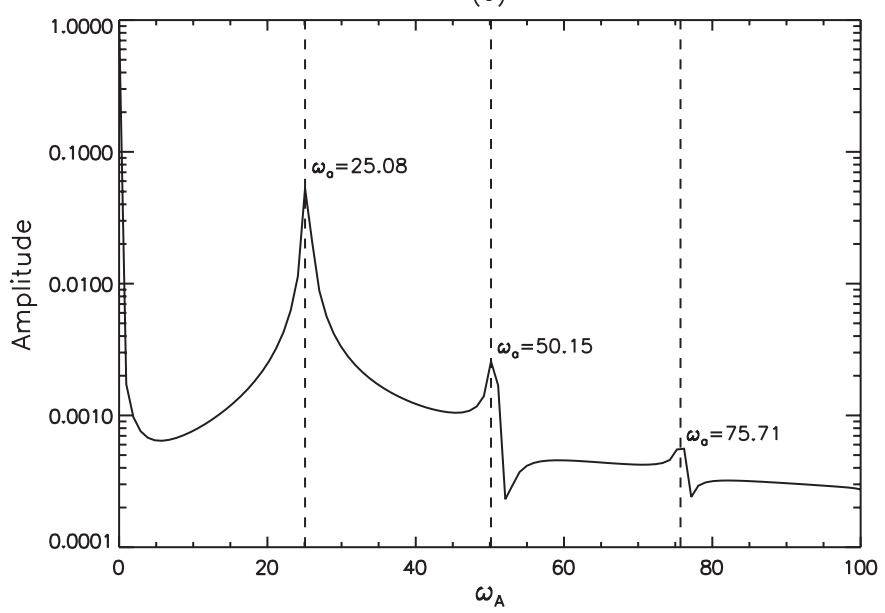

FIG. 8. (a) Time dependence of the magnetic field amplitude for $\mathrm{Rm}=70$ and $a=0.62$. (b) Fourier spectrum for the case $\omega_{\mathrm{v}}=14.9$ (after eliminating the exponential decay). The various peaks denote the modulation frequency of the field amplitude $\omega_{\mathrm{a}} \approx 25.08$ (and its overtones).

opposite to the azimuthal flow of the dominant cell. These cases are denoted by negative frequencies.

The temporal development of the amplitude of the dominant eigenmode for two different vortex drift frequencies, but similar Rm, is shown in Fig. 8 (dashed curve: $\omega_{\mathrm{v}} \approx 2.5$; solid curve: $\omega_{\mathrm{v}} \approx 14.9$ ). Both curves show completely different behavior: For $\omega_{\mathrm{v}} \approx 14.9$ the solution decays and its amplitude is modulated with a frequency of $\omega_{\mathrm{a}} \approx 25.08$ (solid curve), whereas at $\omega_{\mathrm{v}} \approx 2.5$ the field amplitude does not show any time dependence except the exponential growth (dashed curve). Regardless of the distinct temporal behavior for both cases the field structure is quite similar (except the pulsating character in the modulated case) and remains close to the pattern already observed in the previously discussed unperturbed configuration (Fig. 9).

The occurrence of nonaxisymmetric solutions with oscillating energy is a clear indication for the presence of two distinct azimuthal modes with the same growth rate (i.e., real part of the eigenvalues) but with different frequencies (imaginary part of eigenvalues). In fact, simulations that cover a sufficient number of periods exhibit characteristic beat patterns, indicating that the frequencies of the superimposed modes are very close.
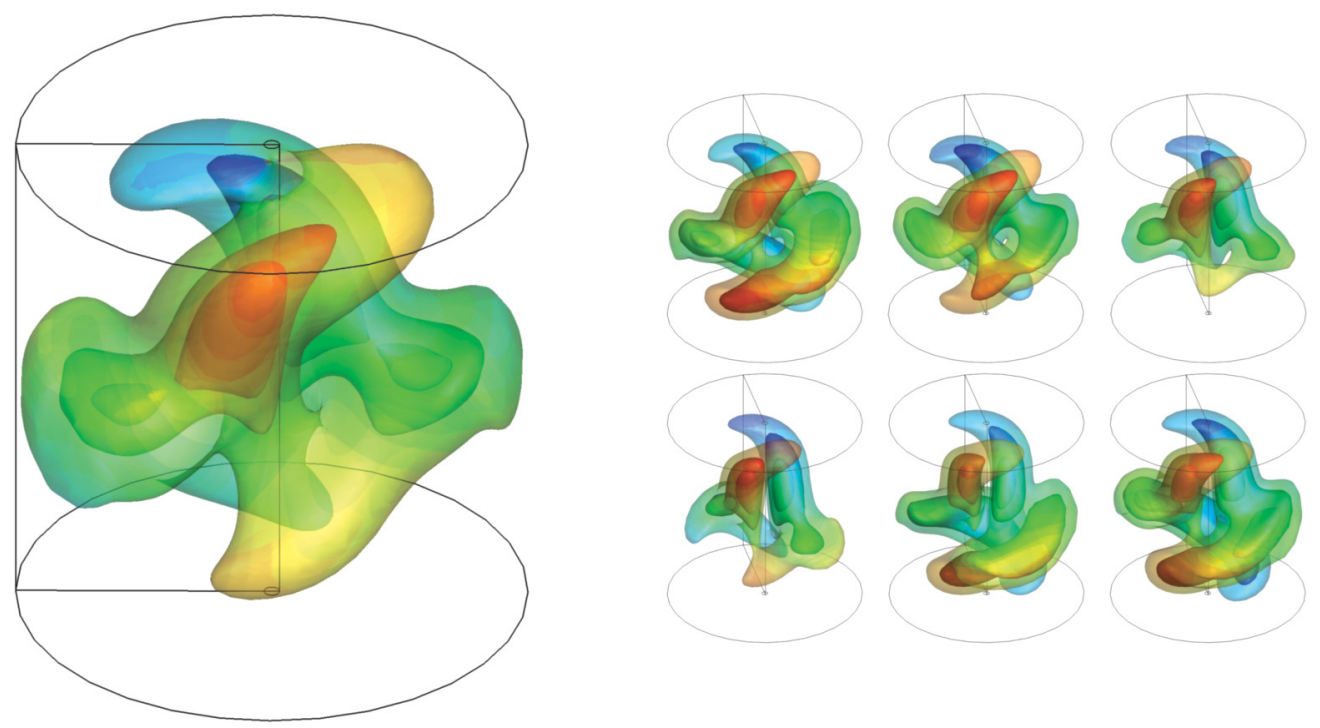

FIG. 9. (Color online) Left: Field structure in the resonant regime $\left(\mathrm{Rm}=70, a=0.62, \omega_{\mathrm{v}}=2.5\right.$, corresponding to the dashed curve in the left panel of Fig. 8). Right: Time series of a typical solution with amplitude modulation ( $\mathrm{Rm}=70, a=0.62, \omega_{\mathrm{v}} \approx 14.9$, corresponding to the solid curve in the left panel of Fig. 8). The isosurfaces show the magnetic energy density at $10 \%, 30 \%$, and $50 \%$ of the respective maximum value and the colored (gray shaded) contours denote the azimuthal magnetic field $B_{\varphi}$. The time series covers one modulation period with each snapshot scaled by the respective maximum so the effects of exponential decay or growth and the amplitude modulations are eliminated. The period of the amplitude modulation is much shorter than the time scale of the magnetic field drift so the phase of the eigenmode (i.e., its orientation in space) remains nearly constant. 
(a)

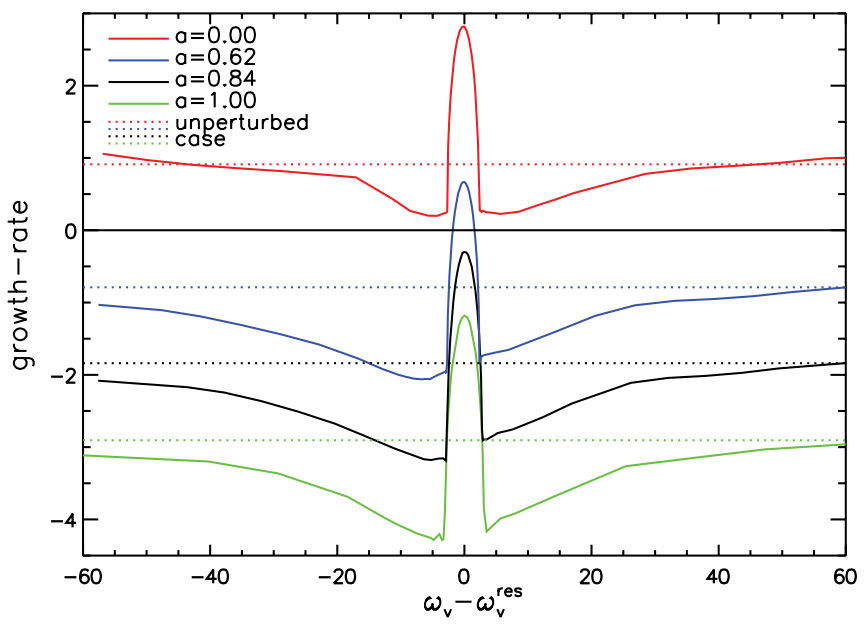

(b)

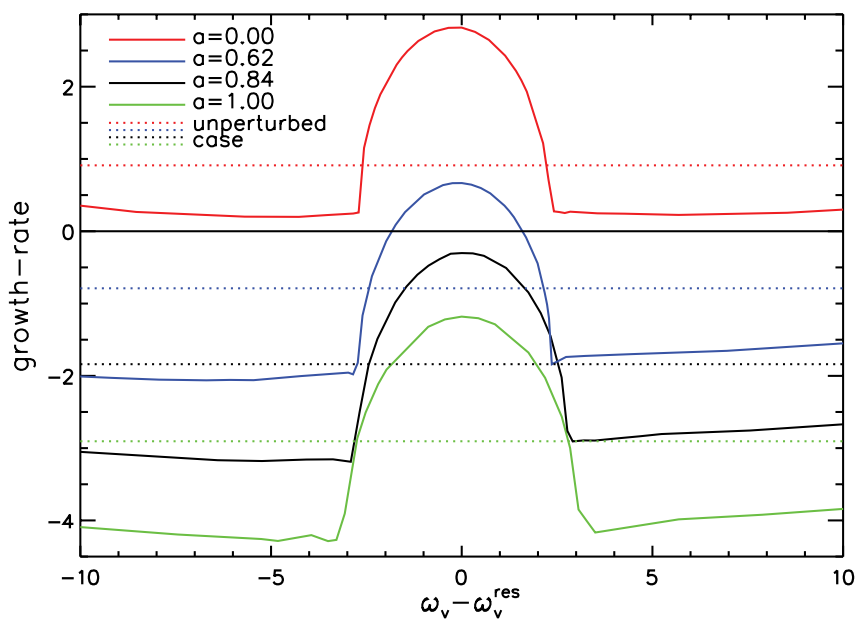

FIG. 10. (Color online) (a) Growth rate versus vortex drift frequency for $\mathrm{Rm}=70$ and $V_{\mathrm{m}}=0.3 U^{\text {max }}$. In the abscissa the resonance frequency $\omega_{\mathrm{v}}^{\text {res }}$ has been subtracted so all curves are centered at the origin. The horizontal dotted lines denote the growth rates without nonaxisymmetric perturbation. (b) Enlarged section of the resonant regime. The growth rates in the resonant regime correspond to nonoscillating $m=1$ eigenmodes, whereas outside of this window the solutions exhibit a modulation of the field amplitude [see also Fig. 11(c)].

We observe field amplitude modulations independently of the degree of equatorial symmetry breaking for a broad range of vortex drift frequencies, whereas unmodulated solutions only occur within a narrow window of relatively slow vortex drift frequencies. The unmodulated solutions are further characterized by a sharp maximum for the corresponding growth rates (Fig. 10), typical for parametric resonance. Characteristic properties of the resonant regime and a comparison to values in the unperturbed state are denoted in Table I. The location of the resonance maximum (denoted by $\omega_{\mathrm{v}}^{\mathrm{res}}$ ) is roughly determined by the field drift frequency of the magnetic eigenmode in the unperturbed case (from now labeled with $\omega_{\mathrm{f}}^{0}$ ). However, a constant gap is observed in runs with equatorial symmetry breaking $(a>0)$, resulting in $\omega_{\mathrm{v}}^{\mathrm{res}} \approx\left(\omega_{\mathrm{f}}^{0}+0.5\right)$, whereas the case $a=0$ remains a particular case with $\omega_{\mathrm{v}}^{\text {res }}=0$. The shift of the resonance frequency with respect to the fundamental frequency in the unperturbed problem probably follows from the interaction of vortex structure and drifting eigenmode similar to the shift of the resonance frequency in periodically forced mechanical systems with damping. Outside of the resonance, the growth rate is suppressed compared to the unperturbed case (denoted by the dotted horizontal line in Fig. 10). Only for very large drift velocities is the value of the purely axisymmetric case obtained again, i.e., the impact of the vortices vanishes when their drift frequency becomes too large. The width of the resonant regime slightly increases with the symmetry-breaking parameter $a$ and so does the relative enhancement of the growth rates and, accordingly, the relative reduction of the critical $\mathrm{Rm}$.

The periodic distortion caused by the drifting vortices also influences the drift behavior of the magnetic eigenmode. In the resonant regime the dynamo eigenmode exhibits an azimuthal field drift that is immediately locked to the vortex drift frequency, i.e., the magnetic field pattern follows the vortices $\left[\omega_{\mathrm{f}} \sim \omega_{\mathrm{v}}\right.$, Fig. 11(b)]. This frequency locking also applies without equatorial symmetry breaking $(a=0)$, which does not show any field drift without vortices [red (topmost) curve in Fig. 11(b)]. Besides the temporal decay and growth, the (constant) azimuthal field drift determines the only time scale in the resonant regime, whereas outside of the resonant window two different time scales appear: an average azimuthal field drift that is roughly determined by the equatorial symmetrybreaking parameter $a$ [solid curves in Fig. $11(a)^{1}$ and the

\footnotetext{
${ }^{1}$ This does not hold in the vicinity of the transition between the modulated regime and the resonant regime where the field drift exhibits a divergent trend.
}

TABLE I. Characteristics properties of the resonant window for different values of the equatorial symmetry breaking $a$. All data stems from runs with $\mathrm{Rm}=70$. The critical magnetic Reynolds number (columns 6 and 7) has been estimated from inter-/extrapolation of simulation runs with $\mathrm{Rm}=50,70$, and 90 .

\begin{tabular}{lccccccc}
\hline \hline & $\begin{array}{c}\text { Maximum } \\
\text { growth } \\
\text { rate }\end{array}$ & $\omega_{\mathrm{v}}^{\text {res }}$ & $\begin{array}{c}\text { Unperturbed } \\
\text { growth } \\
\text { rate }\end{array}$ & $\omega_{\mathrm{f}}^{0}$ & $\begin{array}{c}\mathrm{Rm}^{\mathrm{c}} \\
\text { without } \\
\text { vortex }\end{array}$ & $\begin{array}{c}\mathrm{Rm}^{\mathrm{c}} \\
\text { at } \\
\text { resonance }\end{array}$ & $\begin{array}{c}\text { Relative } \\
\text { reduction } \\
\text { of Rm }^{\mathrm{c}}\end{array}$ \\
\hline 0.00 & +2.817 & -0.00 & +0.912 & 0.00 & 59.7 & 50.5 & $\begin{array}{c}\text { Width of } \\
\text { unmodulated } \\
\text { regime } \Delta \omega_{\mathrm{v}}\end{array}$ \\
0.62 & +0.667 & -1.99 & -0.798 & -2.63 & 78.5 & 64.8 & $17.4 \%$ \\
0.84 & -0.302 & -2.91 & -1.839 & -3.41 & 92.1 & 72.9 & $20.8 \%$ \\
1.00 & -1.181 & -3.50 & -2.905 & -4.04 & 108.5 & 82.7 & $23.8 \%$ \\
\hline \hline
\end{tabular}


(a)

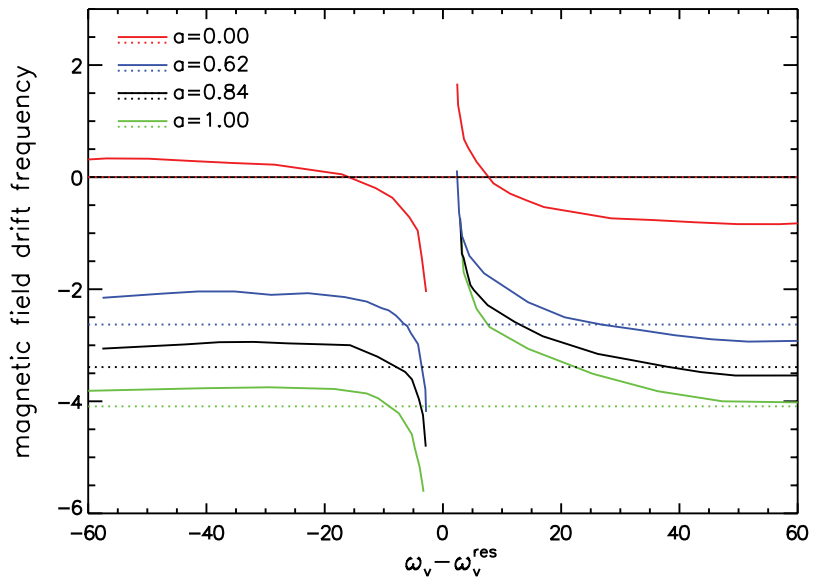

(b)

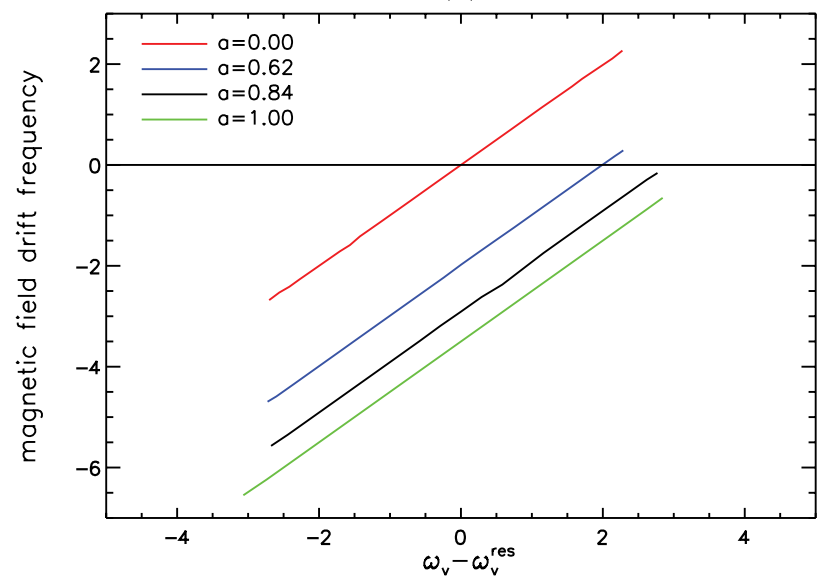

(c)

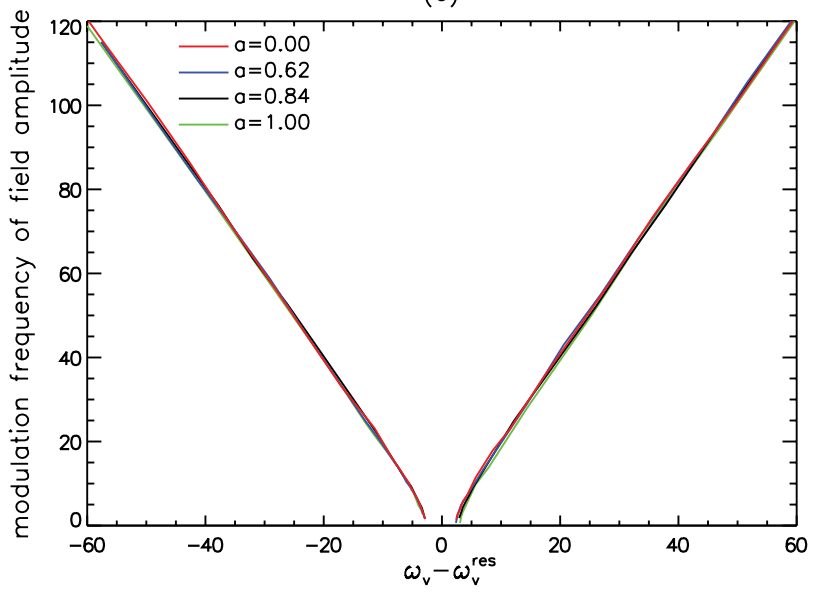

FIG. 11. (Color online) Panels (a) and (b) show the drift frequency of the magnetic eigenmode which is computed from the timederivative of the azimuthal phase $\partial \varphi / \partial t$. (a) average field drift frequency versus the vortex drift frequency for various values of the symmetry-breaking parameter $a$ (value of $a$ increases from top to bottom curves). The solid curves denote the average field drift in the modulated regime. The horizontal dotted lines denote the field drift in the unperturbed case $\left(\omega_{\mathrm{f}}^{0}\right)$. (b) field drift frequency in the resonant regime. The field drift is synchronized with the vortex drift: $\omega_{\mathrm{f}} \sim \omega_{\mathrm{v}}$. (c) Frequency of the magnetic field amplitude modulation for various values of the symmetry-breaking parameter $a$. The frequencies of the amplitude modulation scale $\omega_{\mathrm{a}} \sim 2 \omega_{\mathrm{v}}$. aforementioned modulation of the field amplitude which scales $\sim 2 \omega_{\mathrm{v}}$ [Fig. 11(c)]. In the modulated regime, the field drift is not constant but varies regularly with the same time scale as the amplitude modulation. A movie that shows the time development of four characteristic runs (no vortex, resonant run with frequency locking, and modulated case with positive and/or negative vortex drift frequency) can be found in the supplemental material for this article [45].

In order to illustrate the behavior of the eigenmode in the vicinity of the transition between the resonant and the modulated regime, we have combined the curves shown in Fig. 11 into one drawing. Figure 12 shows a combination of frequencies of the magnetic eigenmode $\left(\omega_{\mathrm{f}} \pm \omega_{\mathrm{a}}\right.$ and $\omega_{\mathrm{f}}$, left panel) and the related growth-rates (right panel) against $\omega_{\mathrm{v}}-\omega_{\mathrm{v}}^{\text {res }}$. By using the sum and difference of the observed frequencies (drift and amplitude modulation), a continuous transition but with a jump in the derivative is achieved between modulated and resonant regime. The abrupt transition from the modulated regime with $\omega_{\mathrm{a}} \sim 2 \omega_{\mathrm{v}}$ to an unmodulated regime with $\omega_{a}=0$ and $\omega_{\mathrm{f}} \sim \omega_{\mathrm{v}}$ occurs when two different eigenmodes merge. At these points, known as exceptional points [29], the eigenvalues of two eigenmodes coincide. In our case, the change of the temporal behavior of the eigenmode results from the merging of the imaginary parts of the two eigenvalues, whereas the real parts (growth rates) are identical in the modulated regime, giving rise to the field amplitude modulation. In the resonant regime (where the field amplitude modulation vanishes) the real parts of the eigenvalues of both interacting modes split and presumably form a "bubble" similar to the behavior of periodical perturbed resonant mechanical systems (see discussion below). In addition, at the exceptional point the two previously linearly independent eigenfunctions collapse and become indistinguishable. Mathematically, this is described by the formation of a nondiagonal Jordan block structure in the matrix representation of the (non-self-adjoint) dynamo operator associated with algebraic eigenvectors [46]. A characteristic property of exceptional points with two coinciding eigenvalues is reflected in the time dependence of the field amplitude, which, exactly at the exceptional point, exhibits an additional secular term linear in $t$ [47] so

$$
\boldsymbol{B}(t) \sim\left(\boldsymbol{B}_{1}+\boldsymbol{B}_{2} t\right) e^{\gamma t} .
$$

Such a time dependence has been found experimentally in an examination of a two-level system in microwave billiards [48] and it would be interesting to identify this term in our simulations. However, relation (9) is exactly valid only very close to the transition point between the modulated and resonant regimes and it seems that, in practice, a unique decomposition of the "measured" growth rates (obtained from our time-stepping scheme) according to Eq. (9) remains impossible. Hence, we can only speculate on the nature of the interacting eigenmodes because, unfortunately, with our present time-stepping method, we can only identify the leading eigenmode, whereas the identification of the second mode would require the use of an appropriate eigenvalue solver. However, it is rather suggestive to assume that the leading mode essentially consists of a dominant $(m=1)$ component with some slight addition of a $(m=2)$-vortex-induced $(m=3)$ component and that the second eigenmode is dominated by a $(m=3)$ component, to which a slight 
(a)

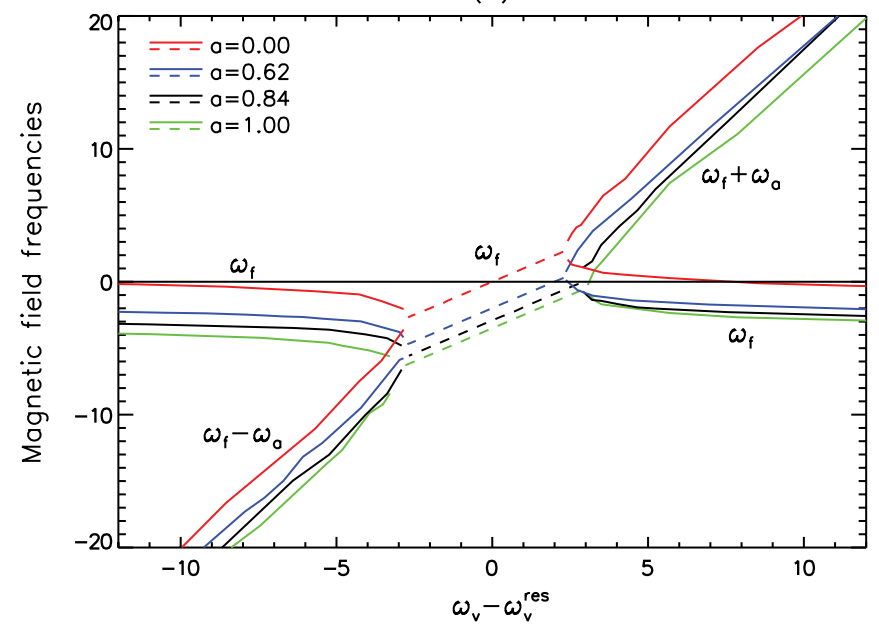

(b)

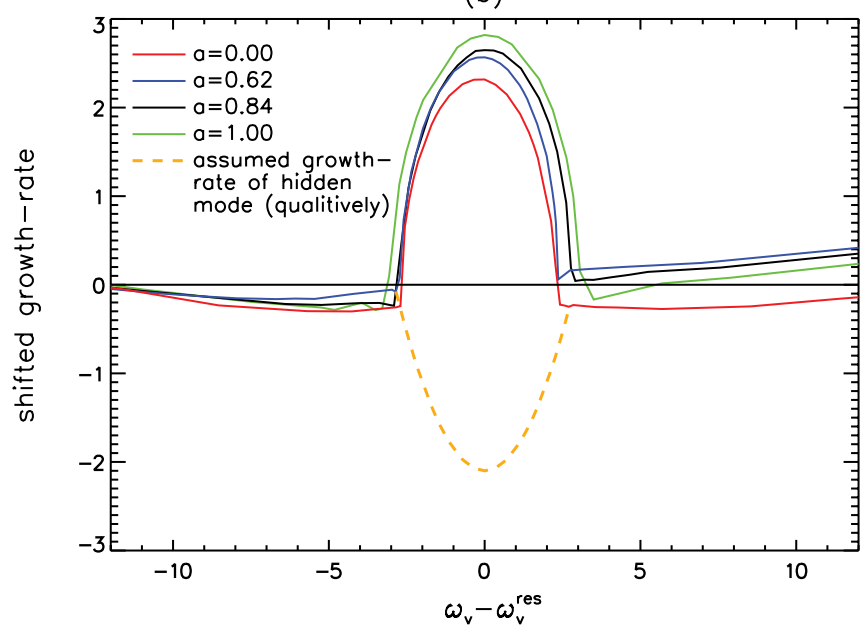

FIG. 12. (Color online) (a) Compilation of frequencies of the magnetic eigenmode versus the vortex drift frequency minus the resonance frequency. The solid curves show a combination of field drift frequency and amplitude modulation frequency and the dashed central part denotes the field drift frequency locked to the vortices in the resonant regime. (b) corresponding growth rates, Note the dashed curve in the bottom part which represents the assumed growth rates of a hidden mode that cannot be identified with the applied time-stepping approach.

$(m=2)$-vortex-induced $(m=1)$ component is added. Independently, we expect that the growth rates of this "hidden" mode qualitatively behave in the same way as in comparable mechanical systems, forming a bubble in the resonant regime. The assumed development has been added in Fig. 12 in terms of the dashed orange curve.

A very similar spectral pattern is well known from mechanical systems subject to periodic perturbations, which, in the undamped case, can often be described by a Mathieu-like equation,

$$
\ddot{x}+\omega_{0}^{2}[1+2 \epsilon \cos (\tilde{\omega} t)] x=0,
$$

where $x$ denotes an oscillating state, $\omega_{0}$ is the fundamental frequency of the system, and $\epsilon$ is the amplitude of the periodic perturbation with frequency $\widetilde{\omega}$. An approximate solution of Eq. (10) can be given in the form of

$$
x(t)=\left[a_{0} \cos \left(\frac{\widetilde{\omega}}{2} t\right)+b_{0} \sin \left(\frac{\widetilde{\omega}}{2} t\right)\right] e^{\mu t} .
$$

Applying some further simplifications the exponent $\mu$ can be calculated as

$$
\mu \approx \pm \frac{1}{2} \sqrt{\left(\epsilon \omega_{0}\right)^{2}-\left(\widetilde{\omega}-2 \omega_{0}\right)^{2}} .
$$

Exponential growth is obtained when the real part of $\mu$ does not vanish so the unstable regime is restricted to the interval $(2-\epsilon) \omega_{0} \leqslant \widetilde{\omega} \leqslant(2+\epsilon) \omega_{0}$. In this regime, exponentially growing solutions occur with a frequency determined by $\widetilde{\omega} / 2$. Outside this resonant regime $\mu$ is purely imaginary and the frequency of the solution is approximately given by

$$
\omega \approx \frac{1}{2} \widetilde{\omega} \pm \sqrt{\left(\widetilde{\omega}-2 \omega_{0}\right)^{2}-\left(\epsilon \omega_{0}\right)^{2}} .
$$

Growth rates and frequencies as obtained from Eqs. (12) and (13) are presented in Fig. 13 and qualitatively show a good agreement with the pattern obtained in our simulations. A comparable pattern is also observed in the eigenvalue spectrum of oscillating $\alpha^{2}$ dynamos with nontrivial radial distribution of $\alpha[49,50]$. However, in those models, the behavior of the growth rates and the frequencies is exchanged: The real part of the eigenvalues merge or split (in the same manner as the frequencies in the left panel of Fig. 12) and the imaginary parts form a bubble corresponding to a restricted regime with oscillatory (axisymmetric) solutions with two conjugate complex eigenvalues.

It is further important to state that the simple model given by Eq. (10) and the corresponding solution (11) can only serve as an analogy for our three-dimensional problem. In particular, our model exhibits dynamo action without perturbation (when $\mathrm{Rm}$ is large enough), whereas the solutions of Eq. (10) only show an instability within a restricted regime. Nevertheless, the resulting frequency behavior denoted by the dotted curves in Fig. 13 is very close to the patterns found in our study (see Fig. 12).

Interestingly, in a few simplified cases dynamo problems were already successfully reduced to a Mathieu-like equation.

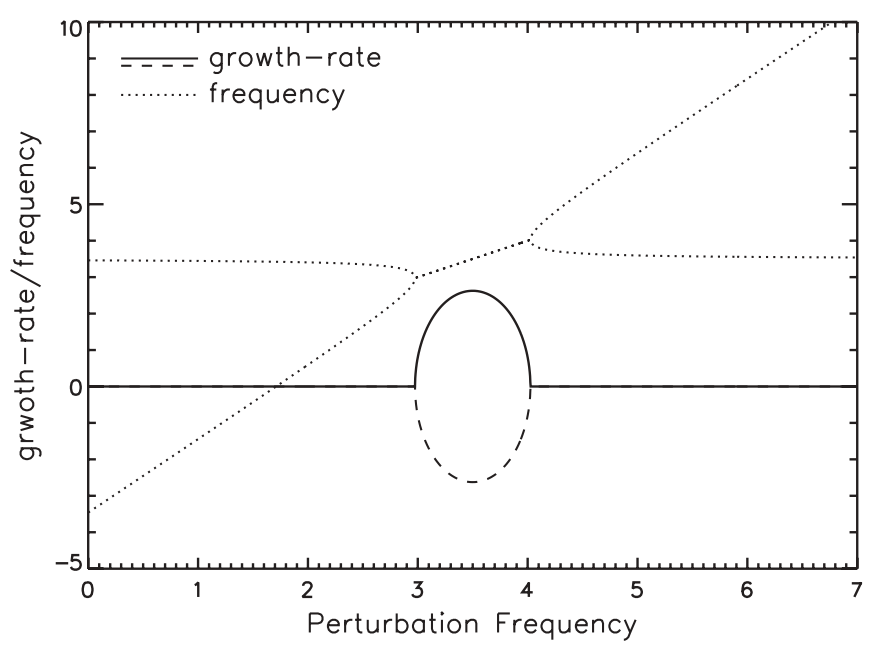

FIG. 13. Growth rates and frequencies of an (approximate) solution to the Mathieu equation (10) versus the perturbation frequency $\widetilde{\omega}$. 
For example, the thin disk approximation used in the galactic $\alpha \omega$ model from Ref. [33] allows a description of the amplitude of the magnetic vector potential by an equation similar to Eq. (10). A parametric resonance (also called swing excitation) is observed when the frequency of the perturbing velocity pattern is twice the natural frequency of the dynamo. Likewise, parametric resonances have been found in a Bullard-type disk dynamo model with a periodic modulation of the disk rotation. The model is based on a damped variant of Eq. (10) and resonances which allow magnetic field excitation for rather low velocities of the conducting disk are also observed at higher frequencies [51]. However, a corresponding reduction of our complicated three-dimensional dynamo to a simplified model such as in Eq. (10) must be left for future work.

\section{CONCLUSIONS}

We have examined kinematic dynamo action of a von Kármán-like flow of a conducting fluid in a cylindrical container. When the flow breaks the ideal equatorial symmetry of the system, the critical magnetic Reynolds number for the onset of dynamo action increases with the amount of symmetry breaking and a time dependence is introduced in terms of an azimuthal drift motion of the dominant dynamo eigenmode. The frequency of this drift increases with the amount of symmetry breaking as well as with the magnetic Reynolds number.

The main focus of our examinations has been on the interaction of this field drift with a nonaxisymmetric timedependent velocity perturbation and the resulting impact on dynamo action. In summary, what we observe is the following: The temporal behavior of the system is governed by three different time scales: the decay/growth time, the magnetic field drift, and the vortex drift, which, in contrast to the first two, represents an imposed quantity. For rather slow vortex drift frequencies, the first magnetic eigenmode (dominated by an $(m=1)$ component $)$ is enslaved by the drifting vortex pattern, hence, we see here $\omega_{\mathrm{f}} \sim \omega_{\mathrm{v}}$. Connected with the linear frequency relationship in this regime we observe a parabolic shape of the growth rate, with a maximum close to the resonance point where the vortex drift frequency would roughly correspond to the eigenfrequency of the magnetic field for the unperturbed, axisymmetric flow, and a quadratic reduction of the growth rate nearby this point. Outside of the resonant regime the field amplitude and the field drift are modulated with twice the frequency of the vortex drift. Phenomenologically, the development of growth rates and frequencies can be described by a Mathieu-like equation.

The observed behavior can also be explained on the basis of simple physical principles. For sufficiently slowly drifting vortices the system adjusts itself to an optimum state and the (azimuthal) phase between magnetic eigenmode and vortex pattern remains fixed so that the field growth becomes maximal. This state is essential characterized by the alignment between the magnetic eigenmode and the nonaxisymmetric velocity mode which is roughly the same independently of symmetry breaking or vortex drift (see Fig. 14). Increasing $\omega_{\mathrm{v}}$, the magnetic eigenmode cannot follow the ever faster $(m=2)$ vortex drift, but "bethinks" of its own eigenvalue (in the unperturbed state) to which it converges in the limit $\left|\omega_{\mathrm{v}}\right| \rightarrow \infty$. In doing so, it will be "beaten" by the $m=2$ vortex mode with an ever-increasing frequency $2 \omega_{\mathrm{v}}$, which explains the occurrence of the second frequency involved.

The simulations presented in this study show similarities with the results from Ref. [16] where dynamo action was examined using a flow field obtained from nonlinear simulations of spherical $s 2 t 2$ flow in a sphere. Kinematic dynamo simulations

$$
a=0.00
$$
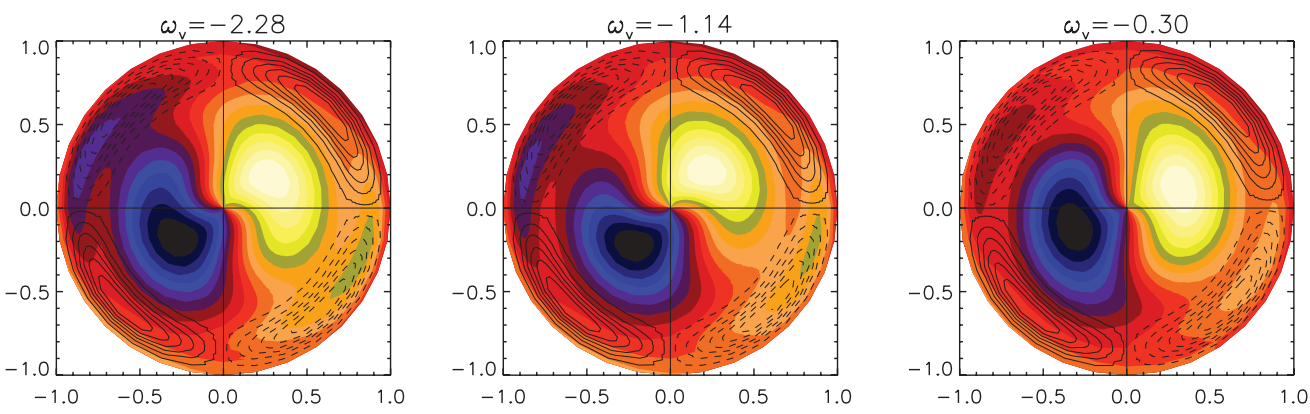

$$
a=0.62
$$
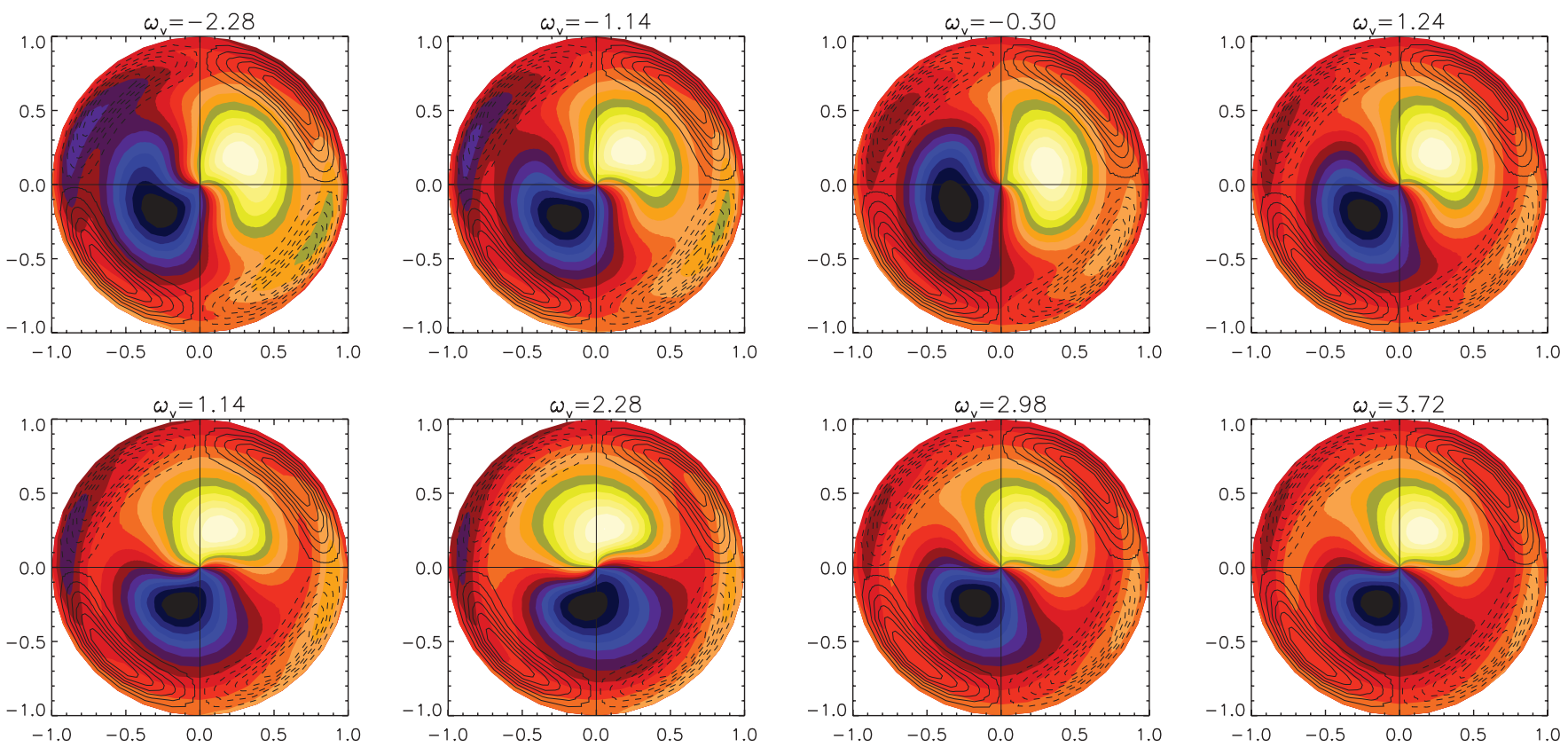

FIG. 14. (Color online) Alignment of magnetic eigenmode and velocity perturbation for different values of the vortex drift frequency $\omega_{\mathrm{v}}$. All runs stem from the resonant regime. The color-coded (gray shaded) structure denotes $B_{\varphi}$ and the contour lines show the axial velocity perturbation $u_{z}^{\mathrm{v}}$. Left: $a=0$; right: $a=0.62$. 
using the time-averaged flow or different snapshots of the velocity field did not exhibit dynamo action, whereas this was indeed the case when considering the time-dependent flow. The exclusive occurrence of dynamo action with a time-dependent flow was interpreted as dynamo action based on non-normal growth originally described in Refs. [26,27]. Looking at the details, a number of differences become visible between the study of Ref. [16] and the model presented here. The key result of Ref. [16] is a magnetic field amplification induced by two counterpropagating $m=2$ waves that were anchored at the poles of a sphere. However, in their study the authors did not find any clear sharp resonant-like regime. Furthermore, they only found a more complex temporal (i.e., cyclic) behavior in one special case with a coupled Navier-Stokes and induction equation which leads to a phase jump in the alignment between the nonaxisymmetric velocity mode and the magnetic eigenmode enforced by the back-reaction of the Lorentz force on the velocity field. In all other runs presented in Ref. [16] the orientation of the magnetic eigenmode remains fixed in space and time and no azimuthal drift is observed (this behavior is similar to the results presented in Ref. [27], where only real eigenvalues of the leading eigenmodes were obtained). The most probable explanation for the distinct behavior results from the differences in the hydrodynamic base of the model of Ref. [16], which does not show any equatorial symmetry breaking (thus, the basic magnetic state is stationary and non-drifting). Moreover, the wavelike distortions in Ref. [16] consist of two counterpropagating $m=2$ patterns that were anchored at the poles of a sphere which most probably inhibits the phase-locking phenomenon observed in our study. As a further difference, we observe a resonant behavior even in runs without equatorial symmetry breaking and with stationary vortices, i.e., in systems without any time dependence so no time-dependent contribution is available that may provide for a mixture of non-normal modes. Hence, the role of non-normal growth in our models and the comparability with the model of Ref. [16] must for now remain an open question.

It is difficult to conclude if the resonance effect can be realized in existing dynamo experiments. Although a coincidence of forcing frequency (the frequency of the drift motion) and magnetic field frequency cannot be ruled out, the resonance condition is a quite particular case and most probably can only be fulfilled by chance. This is particularly true in the case of a more realistic nonlinear analysis, in which the Navier-Stokes and the induction equation are coupled so the drift of the vortex pattern might strongly be influenced by back-reaction of the magnetic field. Furthermore, in the examined VKS-like configuration, the vortex drift frequencies observed in the water experiment are far away from the resonance condition (at least for reasonable values of the equatorial symmetry breaking) so it is unlikely that the vortices affect the VKS dynamo in its actual setup. Nevertheless, it might be suggestive to change the large-scale flow geometry in order to adjust the relation of vortex drift and field drift, e.g., by changing the aspect ratio [52] or by fixing the vortices using some wall inhomogeneity. From Table I it follows immediately that the most promising state to realize the resonance in the experiment would be in a configuration that suppresses the equatorial symmetry breaking (e.g., using a ring in the equatorial plane). In that case, the resonance maximum occurs for nondrifting vortices $\left(\omega_{\mathrm{v}}=0\right)$, providing a reduction of $\sim 15 \%$ (from $\mathrm{Rm}^{\mathrm{c}}=59.7$ to $\mathrm{Rm}^{\mathrm{c}}=50.5$ ). This configuration is definitely achievable in the experiment where the vortices can be anchored by mounting some inhomogeneity on the outer cylinder wall like, e.g., holes or fingers. However, even when the resonance condition theoretically is adjusted, it remains unclear whether a parametric resonance condition can be fulfilled because in the highly turbulent regime the vortex position undergoes considerable fluctuations. These fluctuations (which indeed are observed in the water experiment) could be modeled by introducing a random phase in the equations for the nonaxisymmetric flow perturbation but, in contrast to amplitude fluctuations, such phase noise is known to prevent the occurrence of a parametric resonance [53].

\section{ACKNOWLEDGMENTS}

A.G. and F.S. acknowledge helpful discussions with Oleg Kirillov and financial support from Deutsche Forschungsgemeinschaft (DFG) in frame of the Collaborative Research Center (SFB) 609. J.B. is grateful for the support of the Spanish government through Contract No. FIS2011-24642. The authors thank B. Knaepen and D. Carati of the Université Libre de Bruxelles for inviting them to the Brussels Summer program 2009. The computations were performed using GPUs provided by the CUDA Research Center at TU Dresden (now CUDA Center of Excellence [54]).
[1] F. Stefani, A. Gailitis, and G. Gerbeth, Z. Angew. Math. Mech. 88, 930 (2008).

[2] A. Gailitis, O. Lielausis, S. Dement'ev, E. Platacis, A. Cifersons, G. Gerbeth, T. Gundrum, F. Stefani, M. Christen, H. Hänel, and G. Will, Phys. Rev. Lett. 84, 4365 (2000).

[3] R. Stieglitz and U. Müller, Phys. Fluids 13, 561 (2001).

[4] R. Monchaux, M. Berhanu, M. Bourgoin, M. Moulin, P. Odier, J.-F. Pinton, R. Volk, S. Fauve, N. Mordant, F. Pétrélis, A. Chiffaudel, F. Daviaud, B. Dubrulle, C. Gasquet, L. Marié, and F. Ravelet, Phys. Rev. Lett. 98, 044502 (2007).

[5] M. L. Dudley and R. W. James, Proc. R. Soc. London A 425, 407 (1989).
[6] P. Odier, J.-F. Pinton, and S. Fauve, Phys. Rev. E 58, 7397 (1998).

[7] C. B. Forest, R. A. Bayliss, R. D. Kendrick, M. D. Nornberg, R. O'Connell, and E. J. Spence, Magnetohydrodynamics 38, 107 (2002).

[8] L. Marié, J. Burguete, F. Daviaud, and J. Léorat, Eur. Phys. J. B 33, 469 (2003).

[9] M. Bourgoin, R. Volk, P. Frick, S. Khripchenko, P. Odier, and J.-F. Pinton, Magnetohydrodynamics 40, 3 (2004).

[10] F. Stefani, M. Xu, G. Gerbeth, F. Ravelet, A. Chiffaudel, F. Daviaud, and J. Léorat, Eur. J. Mech. B 25, 894 (2006).

[11] F. Ravelet, A. Chiffaudel, F. Daviaud, and J. Léorat, Phys. Fluids 17, 7104 (2005). 
[12] M. Berhanu, R. Monchaux, S. Fauve, N. Mordant, F. Pétrélis, A. Chiffaudel, F. Daviaud, B. Dubrulle, L. Marié, F. Ravelet, M. Bourgoin, P. Odier, J.-F. Pinton, and R. Volk, Europhys. Lett. 77, 59001 (2007).

[13] C. Gissinger, A. Iskakov, S. Fauve, and E. Dormy, Europhys. Lett. 82, 29001 (2008).

[14] C. Gissinger, E. Dormy, and S. Fauve, Phys. Rev. Lett. 101, 144502 (2008).

[15] K. Reuter, F. Jenko, and C. B. Forest, New J. Phys. 11, 013027 (2009).

[16] K. Reuter, F. Jenko, A. Tilgner, and C. B. Forest, Phys. Rev. E 80, 056304 (2009).

[17] R. Monchaux, M. Berhanu, S. Aumaître, A. Chiffaudel, F. Daviaud, B. Dubrulle, F. Ravelet, S. Fauve, N. Mordant, F. Pétrélis, M. Bourgoin, P. Odier, J.-F. Pinton, N. Plihon, and R. Volk, Phys. Fluids 21, 035108 (2009).

[18] P. H. Roberts, G. A. Glatzmaier, and T. L. Clune, Geophys. Astrophys. Fluid Dyn. 104, 207 (2010).

[19] I. V. Khalzov, B. P. Brown, F. Ebrahimi, D. D. Schnack, and C. B. Forest, Phys. Plasmas 18, 032110 (2011).

[20] E. J. Kaplan, M. M. Clark, M. D. Nornberg, K. Rahbarnia, A. M. Rasmus, N. Z. Taylor, C. B. Forest, and E. J. Spence, Phys. Rev. Lett. 106, 254502 (2011).

[21] A. Giesecke, F. Stefani, and G. Gerbeth, Phys. Rev. Lett. 104, 044503 (2010).

[22] A. Giesecke, C. Nore, F. Stefani, G. Gerbeth, J. Léorat, W. Herreman, F. Luddens, and J.-L. Guermond, New J. Phys. 14, 053005 (2012).

[23] A. de la Torre and J. Burguete, Phys. Rev. Lett. 99, 054101 (2007).

[24] F. Ravelet, A. Chiffaudel, and F. Daviaud, J. Fluid Mech. 601, 339 (2008).

[25] P.-P. Cortet, P. Diribarne, R. Monchaux, A. Chiffaudel, F. Daviaud, and B. Dubrulle, Phys. Fluids 21, 025104 (2009).

[26] E. Dormy and D. Gérard-Varet, Europhys. Lett. 81, 64002 (2008).

[27] A. Tilgner, Phys. Rev. Lett. 100, 128501 (2008).

[28] J. Burguete and A. De La Torre, Int. J. Bifurcation Chaos 19, 2695 (2009).

[29] T. Kato, Perturbation Theory of Linear Operators (Springer, New York, 1966).

[30] M. V. Berry, Czech. J. Phys. 54, 1039 (2004).

[31] J.-S. Chen and D. B. Bogy, J. Appl. Mech. 59, 390 (1992).
[32] R. S. Mackay and P. G. Saffman, Proc. R. Soc. London A 406, 115 (1986).

[33] M. Chiba and M. Tosa, Mon. Not. R. Astron. Soc. 244, 714 (1990).

[34] D. Schmitt and G. Rüdiger, Astron. Astrophys. 264, 319 (1992).

[35] D. Moss, Astron. Astrophys. 308, 381 (1996).

[36] R. Rohde, G. Rüdiger, and D. Elstner, Astron. Astrophys. 347, 860 (1999).

[37] D. Sokoloff and N. Piskunov, Mon. Not. R. Astr. Soc. 334, 925 (2002).

[38] A. de La Torre, J. Burguete, and C. Pérez-García, Eur. Phys. J. ST 146, 313 (2007).

[39] A. Iskakov and E. Dormy, Geophys. Astrophys. Fluid Dyn. 99, 481 (2005).

[40] A. Giesecke, F. Stefani, and G. Gerbeth, Magnetohydrodynamics 44, 237 (2008).

[41] L. Marié, C. Normand, and F. Daviaud, Phys. Fluids 18, 017102 (2004).

[42] C. Gissinger, Europhys. Lett. 87, 39002 (2009).

[43] D. D. Joseph, Stability of Fluid Motions I (Springer, Berlin, 1976).

[44] J. Guckenheimer and P. Holmes, Nonlinear Oscillations, Dynamical Systems and Bifurcations of Vector Fields (Springer, Berlin, 2002).

[45] See Supplemental Material at http://link.aps.org/supplemental/ 10.1103/PhysRevE.86.066303 for a movie that shows the temporal field behavior for unperturbed, resonant, and modulated cases.

[46] U. Günther, F. Stefani, and G. Gerbeth, Czech. J. Phys. 54, 1075 (2004).

[47] O. Kirillov, Fortschr. Phys. (2012), http://dx.doi.org/10.1002/ prop. 201200068.

[48] B. Dietz, T. Friedrich, J. Metz, M. Miski-Oglu, A. Richter, F. Schäfer, and C. A. Stafford, Phys. Rev. E 75, 027201 (2007).

[49] F. Stefani and G. Gerbeth, Phys. Rev. E 67, 027302 (2003).

[50] A. Giesecke, F. Stefani, and G. Gerbeth, Geophys. Astrophys. Fluid Dynam. (2012), doi: 10.1080/03091929.2012.668543.

[51] J. Priede, R. Avalos-Zuñiga, and F. Plunian, Phys. Lett. A 374, 584 (2010).

[52] K. M. Kuzanyan and D. D. Sokoloff, Astrophys. Space Sci. 208, 245 (1993).

[53] F. Pétrélis and S. Fauve, Europhys. Lett. 76, 602 (2006).

[54] CUDA Center of Excellence at TU Dresden, http://ccoedresden.de. 